\title{
Integrated modeling for ion cyclotron resonant heating in toroidal systems
}

\author{
M. Jucker ${ }^{\text {a,* }}$, J.P. Graves ${ }^{\text {a }}$, W.A. Cooper ${ }^{a}$, N. Mellet ${ }^{a}$, T. Johnson ${ }^{b}$, S. Brunner ${ }^{a}$ \\ a Ecole Polytechnique Fédérale de Lausanne (EPFL), Centre de Recherches en Physique des Plasmas, Association EURATOM-Confédération Suisse, CH-1015 Lausanne, Switzerland \\ ${ }^{\mathrm{b}}$ VR-Euratom Association, EES, KTH, Stockholm, Sweden
}

\section{A R T I C L E I N F O}

\section{Article history:}

Received 6 July 2010

Received in revised form 8 December 2010

Accepted 21 December 2010

Available online 24 December 2010

\section{Keywords:}

Integrated modeling

Ion cyclotron

Radio frequency heating

\begin{abstract}
A B S T R A C T
An integrated model capable of self-consistent Ion Cyclotron Resonant Heating (ICRH) simulations has been developed. This model includes both full shaping and pressure effects, warm contributions to the dielectric tensor, pressure anisotropy and finite orbit width. It evolves the equilibrium, wave field and full hot particle distribution function until a self-consistent solution is found. This article describes the workings of the three codes VMEC, LEMan and VENUS and how they are linked for iterated computations in a code package we have named SCENIC. The package is thoroughly tested and it is demonstrated that a number of iterations have to be performed in order to find a consistent solution. Since the formulation of the problem can treat general 3D systems, we show a quasi-axisymmetric stellarator low power test case, and then concentrate on experimentally relevant Joint European Torus (JET) 2D configurations.
\end{abstract}

(C) 2010 Elsevier B.V. All rights reserved.

\section{Introduction}

In present day fusion devices, radio frequency (RF) heating of minority species comprises a fundamental auxiliary heating system. Furthermore, RF heating in the ion cyclotron range of frequencies (ICRH) is considered to be one of the major contributors to additional heating in ITER. The wave-particle interactions between the RF field and the plasma ions depend strongly on the strength of the left-handed component $E^{+}$of the electric field. Direct heating of the bulk plasma is inefficient since $E^{+}$vanishes at the fundamental cyclotron resonance of the majority species. In contrast, minority heating is much more promising: the choice of the minority (charge and mass) determines the location of the cyclotron resonance, which will lie on a different location than the bulk resonance (assuming that the charge-mass ratio is different from the bulk species). The left-handed $E^{+}$component will not vanish at the resonant layer of the minority, and therefore the minority species can be heated efficiently. Coulomb collisions will then assure the heating of the complete plasma. Another application of ICRF is the generation of RF induced current through Ion Cyclotron Current Drive (ICCD). Here, the total current is changed locally for the control of MHD instabilities. Moreover, it has recently been shown [1] that parallel velocity asymmetry in the distribution function, and the radial drift of the fast ions, which result from toroidally propagating $\mathrm{RF}$ waves, can affect MHD stability. We will describe here a numerical package which is capable of retaining such effects.

\footnotetext{
* Corresponding author. Tel.: +41216936536.

E-mail address: martin.jucker@a3.epfl.ch (M. Jucker).
}

Previously, other code suites have been developed for the study of radio frequency heating. For instance, FIDO has been coupled to LION (giving SELFO) [2], TORIC to SSFPQL $[3,4]$ or ORBIT-RF to AORSA [5], but none of these codes take into account the possible change of the equilibrium due to the RF wave-particle interactions. However, applications of ICRF act on the state of the plasma, be it through heating or current drive, and change, at least locally, the equilibrium. This work proposes a 3D self-consistent numerical approach for studying the effects of minority ICRH on all important quantities such as the fast ion distribution function, the wave field and the magnetic equilibrium. The MHD equilibrium represents one part of the iterated scheme, called SCENIC, and this possibility of including the equilibrium within the self-consistent computations of SCENIC represents a complete novelty. Another original feature of SCENIC is its ability to include three-dimensional geometries, since all codes of the package work intrinsically in threedimensional space. Although all constituents have been successfully applied independently to non-axisymmetric plasmas in the past, and SCENIC has been tested for non-axisymmetry as well, we will in this work primarily concentrate on 2D JET-like equilibria. As the toroidal dimension cannot be removed, two-dimensionality is achieved by forcing all parameters to be constant along the toroidal direction in the equilibrium. We will focus on axisymmetric plasmas mostly because such cases are more intuitive and the results of this first application of SCENIC can be verified more easily. Nevertheless, we also illustrate the functionality of the three constituent parts of the model (wave, particle and equilibrium) for a 3D quasi-axisymmetric stellarator. In general, SCENIC has been designed to focus on capturing exotic fast particle physics, and most developmental efforts have therefore concentrated on correctly dealing with highly energetic non-standard particle orbits, 


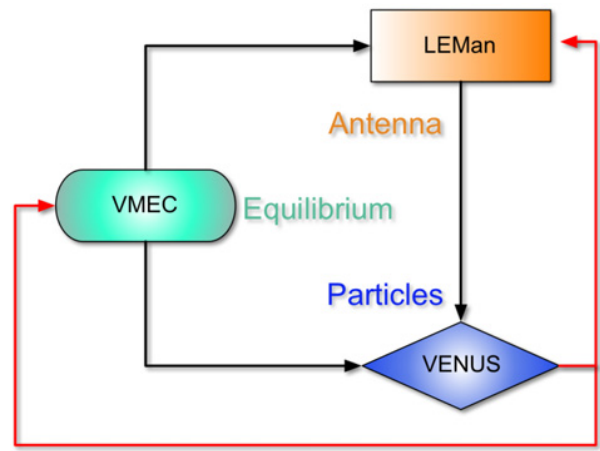

Fig. 1. The code package SCENIC and its components: VMEC provides MHD equilibrium, LEMan the wave field and wave numbers and VENUS advances the distribution function with a full-f scheme. At the end of each iteration, VENUS produces new inputs to VMEC and LEMan for the next iteration.

and their effects on the tail of the distribution function. As a result, the main restrictions of SCENIC lie in the wave code due to zeroth order expansion in Larmor radius. We will here consider helium-3 minority heating in deuterium plasma, in order to avoid second harmonic resonances in the plasma, which are neglected in the wave field computations.

This article will first describe the components and workings of the code package SCENIC in detail (Section 2), including the way the codes are interfaced. Section 3 shows basic numerical tests and studies, before illustrating the complete package in Section 4 . Here, we will show the importance of the iteration scheme and the physics included by applying the code to a JET relevant 2D test case. The article will be concluded in Section 5 .

\section{SCENIC}

The SCENIC (Self-ConsistENt Ion Cyclotron) package is composed of an anisotropic pressure version of the MHD equilibrium solver VMEC [6], the full-wave code LEMan [7,8] and the Monte Carlo guiding centre single particle code VENUS $[9,10]$. All of these codes are set up to handle full 3D plasmas, i.e. capability of dealing with stellarators, magnetic ripple, and others. Here, however, we will only show applications to axisymmetric scenarios, since these results are more intuitive and better understood, and therefore more adapted for a first application of this newly created code package. Fig. 1 shows a schematic view of the workings of the package: VMEC computes an equilibrium based on pressure and current profiles, treating the background distribution Maxwellian (isotropic) and the hot particle distribution bi-Maxwellian (anisotropic). The bi-Maxwellian allows for different temperatures parallel and perpendicular to the magnetic field (the only constraints are that VMEC imposes nested magnetic surfaces with a single magnetic axis, such that magnetic islands and stochastic regions cannot be modelled). Using this equilibrium, LEMan computes the (3D) wave field given a toroidal mode spectrum, frequency and geometry of the antenna. The power deposition is also computed and used later on for the scaling of the electric field, such that the latter corresponds in amplitude to the desired deposited power. VENUS reads the equilibrium, wave numbers and electric field from the two previous codes. It then advances the distribution function of the minority ion species using Monte Carlo collision and quasilinear diffusion operators. At the end of each iteration, the resulting distribution function is integrated (exactly within a guiding centre framework) and the moments needed by VMEC and LEMan are produced for the next iteration. Fig. 2 shows an artist's view of SCENIC, including the magnetic equilibrium, RF wave field and power deposition, all acting on the particle orbits, thus deforming the distribution function of the minority species.

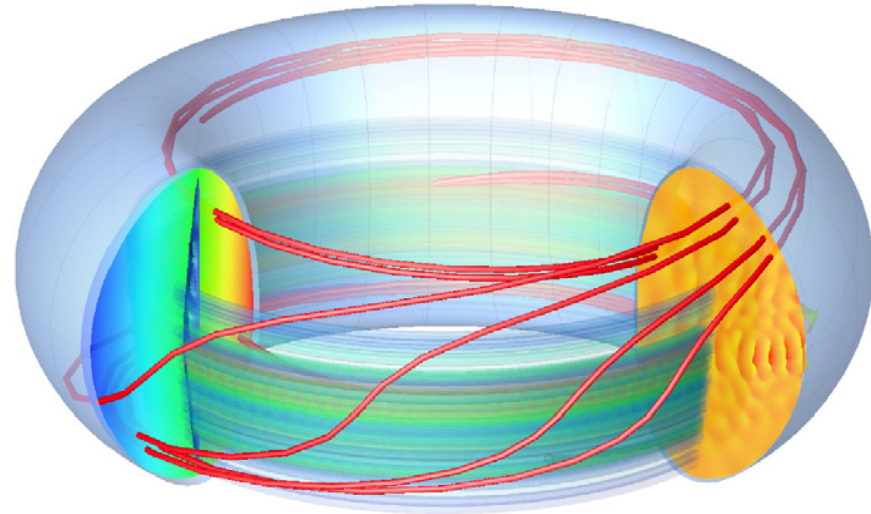

Fig. 2. Artist's view of the SCENIC package. The poloidal cuts on the left and the right show the magnetic field strength (representing the equilibrium) from VMEC together with the 2D power deposition from VENUS, and the wave field from LEMan (shown is $\Re\left(E^{+}\right)$, right) respectively. In three dimensions, a test particle orbit and the power deposition from LEMan are shown.

\subsection{Equilibrium: VMEC}

The MHD equilibrium code VMEC [11] has been extended to include the effects of an anisotropic distribution function for a minority ion species in a thermal Maxwellian plasma. Although simple equivalent Maxwellians are known to have deficiencies in modelling high energy tails [12], it has been shown that a specific form of a bi-Maxwellian can be used as a good analytical approximation of the hot (minority) particle distribution function $[13,14]$. Such a distribution can be written as

$$
\begin{aligned}
F_{h}(s, E, \mu)= & \mathcal{N}(s)\left(\frac{m}{2 \pi T_{\perp}(s)}\right)^{3 / 2} \\
& \times \exp \left[-\frac{\mu B_{c}}{T_{\perp}(s)}-\frac{\left|E-\mu B_{c}\right|}{T_{\|}(s)}\right],
\end{aligned}
$$

where $m$ is the mass of the particle, $E=m v^{2} / 2$ the particle energy, $\mu=m v_{\perp}^{2} / 2 B$ the magnetic moment, $T_{\perp, \|}$ the perpendicular/parallel temperature and $s$ a flux label based on the toroidal flux. More exactly, $s$ denotes the radial position corresponding to a guiding centre average over the orbit of a particle with given $E$ and $\mu . B_{c}$ denotes the resonant layer where the RF heating is applied. The density factor $\mathcal{N}(s)$ is related to the physical density $n_{h}$ through $n_{h}(s, \theta)=\mathcal{N}(s) \sqrt{T_{\|}(s) / T_{\perp}(s)} \mathcal{C}(s, \theta)$, with $n_{h}(s, \theta)$ the hot particle density and $\mathcal{C}(s, \theta)$ a geometric factor described later. It is convenient to use $\mathcal{N}(s)$ rather than the physical density $n_{h}(s, \theta)$, due to the fact that the first can be prescribed as a radial profile while the latter is a function of poloidal angle $\theta$ in 2D geometries, and of the field strength $B$ in three-dimensional plasmas. We will use the 2D notation with $\theta$ throughout this article for a more intuitive understanding. The required input radial profiles are the background and hot particle pressures, anisotropy and either the safety factor or total toroidal current enclosed within each flux surface. Note that we will call "background" the thermal ions and electrons, which are Maxwellian with static profiles during any simulation. Since VENUS computes the RF induced current, the latter is added to the ohmic current and used as input. Also, the rigid wall Fourier amplitudes are imposed for the shaping of the plasma. This extension of VMEC has been described in detail in Ref. [6] and we point the interested reader to that reference for further information.

\subsection{RF wave field: LEMan}

The dielectric tensor in LEMan is of zeroth order in Larmor radius. We are therefore limited to fundamental minority scenarios 
with SCENIC, since then the ion-ion hybrid resonance does not appear, and no mode conversion takes place. In a non-axisymmetric plasma, LEMan is run with a given wave spectrum of different toroidal and poloidal modes, since in that case the toroidal modes are not decoupled. The wave field is excited by a prescribed antenna, which can be delimited in radial, poloidal and toroidal directions. For axisymmetric cases, the toroidal modes decouple and it is simpler to run LEMan separately for each toroidal mode number excited by the antenna (which is then localised in the radial and poloidal directions only). Here, only one toroidal mode per run is considered, but a full spectrum of poloidal modes is still included. Following Refs. [15,16], we have derived the dielectric tensor for the fast particles modelled by the distribution function (1) to zeroth order in both $\epsilon_{e}=\rho / L$ and $\epsilon_{p}=\rho / \lambda_{\perp}$, where $\rho$ is the Larmor radius, $L$ a characteristic equilibrium length scale and $\lambda_{\perp}$ a characteristic wave length of the perturbing electromagnetic field in the direction perpendicular to the equilibrium magnetic field. The expressions are different for $B \geqslant B_{c}$ and $B<B_{c}$ and read

- For $B \geqslant B_{C}$ :

$$
\begin{aligned}
& \mathcal{E}_{n n}=1-\frac{1}{2 \omega} \frac{\sqrt{T_{\|} / T_{\perp}}}{C_{+}} \sum_{k}\left(\tilde{Z}_{1}^{\|}+\tilde{Z}_{-1}^{\|}\right), \\
& \mathcal{E}_{n b}=-\frac{i}{2 \omega} \frac{\sqrt{T_{\|} / T_{\perp}}}{C_{+}} \sum_{k}\left(\tilde{Z}_{1}^{\|}-\tilde{Z}_{-1}^{\|}\right)=-\mathcal{E}_{b n}, \\
& \mathcal{E}_{\|\|}=1+\frac{2}{\left(k_{\|} v_{\| T}\right)^{2}} \frac{\sqrt{T_{\|} / T_{\perp}}}{C_{+}} \sum_{k}\left(\tilde{\omega}_{p}^{2}-\omega \tilde{Z}_{0}^{\|}\right),
\end{aligned}
$$

- For $B<B_{c}$ :

$$
\begin{aligned}
& \mathcal{E}_{n n}=\mathcal{E}_{n n}^{B \geqslant B_{c}}-\frac{1}{2 \omega} \frac{C_{+}-C_{-}}{C_{+} C_{-}} \sum_{k}\left(\tilde{Z}_{1}^{\perp}+\tilde{Z}_{-1}^{\perp}\right), \\
& \mathcal{E}_{n b}=\mathcal{E}_{n b}^{B \geqslant B_{c}}-\frac{i}{2 \omega} \frac{C_{+}-C_{-}}{C_{+} C_{-}} \sum_{k}\left(\tilde{Z}_{1}^{\perp}-\tilde{Z}_{-1}^{\perp}\right)=-\mathcal{E}_{b n}, \\
& \mathcal{E}_{\|\|}=\mathcal{E}_{\|\|}^{B \geqslant B_{c}}-\frac{C_{+}+C_{-}}{C_{+} C_{-}} \sum_{k}\left(\sqrt{\frac{B_{c}-B}{B_{c}}} \tilde{\omega}_{p}^{2}-\omega \tilde{Z}_{0}^{\perp}\right) .
\end{aligned}
$$

Here, $v_{\| T}^{2}=2 T_{\|} / m$ is the fast particle thermal parallel velocity and

$$
\begin{aligned}
& C_{ \pm}=\frac{B_{c}}{B} \pm \frac{T_{\perp}}{T_{\|}}\left(1-\frac{B_{c}}{B}\right), \\
& Z^{S h}(z)=\frac{z}{\sqrt{\pi}} \int_{-\infty}^{\infty} \frac{1}{z-x} e^{-x^{2}} d x, \quad \operatorname{Im} z>0, \\
& \tilde{Z}_{l}^{\|}=\frac{\tilde{\omega}_{p}^{2}}{\omega-l \Omega_{c}} Z^{S h}\left(\frac{\omega-l \Omega_{c}}{k_{\|} v_{\| T}}\right), \\
& \tilde{Z}_{l}^{\perp}=\sqrt{\frac{B_{c}-B}{B_{c}}} \frac{\tilde{\omega}_{p}^{2}}{\omega-l \Omega_{c}} Z^{S h}\left(\sqrt{\frac{B_{c}}{B_{c}-B}} \frac{\omega-l \Omega_{c}}{k_{\|} v_{\| T}}\right),
\end{aligned}
$$

and $\tilde{\omega}_{p}^{2}=q_{k}^{2} \mathcal{N}_{k} / \epsilon_{0} m_{k}$ the plasma frequency of species $k$. The subscripts $n, b$ and $\|$ denote the normal, bi-normal and parallel components relative to the magnetic field. It is worth highlighting a few observations: First of all, one can see that we exactly recover the zeroth order results obtained in Refs. [15] and [16] in the limit where $T_{\perp} / T_{\|} \rightarrow 1$ and $B_{c} \rightarrow 0$ (i.e. $F_{h} \rightarrow F_{M}$ ). However, the additional parameter $B_{c}$ in the distribution function has the effect that, even if we consider the isotropic case $T_{\perp}=T_{\|}$, some of the additional terms for the region $B<B_{c}$ do not vanish and introduce poloidally dependent corrections to the dielectric tensor.
The parameter $B_{c}$ then assures the presence of a localised cyclotron power deposition and the existence of a preferred pitch angle where the turning points of trapped particle orbits lie in the resonant region [13]. Finally, the plasma frequency in expressions (3) does not depend explicitly on the physical density $n_{h}$, but rather the density factor $\mathcal{N}(s)$ defined in the distribution function (1), which can be considerably higher than the physical density if $T_{\perp} / T_{\|} \gg 1$.

\subsection{Particle-in-cell: VENUS}

\subsubsection{Equations of motion}

The equations of motion are based on a Hamiltonian formulation of the guiding centre orbits of charged particles in an electromagnetic field. They have been derived in the frame of Hamiltonian formalism in Refs. [10] and [17]. The equations of motion include a parallel perturbation in the vector potential while still retaining the canonical structure of the variables. Perpendicular fluctuations of the vector potential are under development and VENUS will be able to include them in the near future. We note here the complete equations of motion in the Boozer variables $(s, \theta, \varphi)$ and the parallel gyroradius $\rho_{\|}=m v_{\|} /(e \sigma B)$, where $\sigma=1 / \mu_{0}-\left(p_{\|}-p_{\perp}\right) / B^{2}$ is related to the mirror stability criterion and must always be positive. The equations of motion read

$$
\begin{aligned}
& \dot{s}=+\frac{\mu_{0} I(s)}{D}\left[\left.\frac{\partial \Phi}{\partial \theta}\right|_{s, \varphi, t}+\frac{1}{\gamma}\left(\frac{\mu}{e}+\sigma \tau \frac{e B}{m_{0}} \rho_{\|}^{2}\right) \frac{\partial B}{\partial \theta}\right. \\
& \left.-\left.\frac{e \sigma^{2} B^{2}}{\gamma m_{0}} \rho_{\|} \frac{\partial \Upsilon}{\partial \theta}\right|_{s, \varphi, t}\right] \\
& +\frac{\mu_{0} J(s)}{D}\left[\left.\frac{\partial \Phi}{\partial \varphi}\right|_{s, \theta, t}+\frac{1}{\gamma}\left(\frac{\mu}{e}+\sigma \tau \frac{e B}{m_{0}} \rho_{\|}^{2}\right) \frac{\partial B}{\partial \varphi}\right. \\
& \left.-\left.\frac{e \sigma^{2} B^{2}}{\gamma m_{0}} \rho_{\|} \frac{\partial \Upsilon}{\partial \varphi}\right|_{s, \theta, t}\right] \\
& \dot{\theta}=-\frac{\mu_{0} I(s)}{D}\left[\left.\frac{\partial \Phi}{\partial s}\right|_{\theta, \varphi, t}+\frac{1}{\gamma}\left(\frac{\mu}{e}+\sigma \tau \frac{e B}{m_{0}} \rho_{\|}^{2}\right) \frac{\partial B}{\partial s}\right. \\
& \left.+\left.\frac{e \sigma B^{2}}{\gamma m_{0}} \rho_{\|}^{2} \frac{\partial \sigma}{\partial s}\right|_{B}\right] \\
& +\frac{e \sigma^{2} B^{2}}{\gamma m_{0} D} \rho_{\|}\left[\psi^{\prime}(s)+\left(\rho_{\|}+\Upsilon\right) \mu_{0} I^{\prime}(s)+\left.\mu_{0} I(s) \frac{\partial \Upsilon}{\partial s}\right|_{\theta, \varphi, t}\right] \\
& +\frac{e \sigma^{2} B^{2}}{\gamma m_{0} D} \rho_{\|}\left(\rho_{\|}+\Upsilon\right) \frac{\partial\left(\sigma B_{s}\right)}{\partial \varphi} \\
& -\frac{\sigma B_{s}}{D}\left[\frac{\partial \chi}{\partial \varphi}+\frac{1}{\gamma}\left(\frac{\mu}{e}+\frac{e B}{m_{0}} \sigma \tau \rho_{\|}^{2}\right) \frac{\partial B}{\partial \varphi}-\frac{e \sigma^{2} B^{2}}{\gamma m_{0}} \rho_{\|} \frac{\partial \Upsilon}{\partial \varphi}\right], \\
& \dot{\varphi}=-\frac{\mu_{0} J(s)}{D}\left[\left.\frac{\partial \Phi}{\partial s}\right|_{\theta, \varphi, t}+\frac{1}{\gamma}\left(\frac{\mu}{e}+\sigma \tau \frac{e B}{m_{0}} \rho_{\|}^{2}\right) \frac{\partial B}{\partial s}\right. \\
& \left.+\left.\frac{e \sigma B^{2}}{\gamma m_{0}} \rho_{\|}^{2} \frac{\partial \sigma}{\partial s}\right|_{B}\right] \\
& +\frac{e \sigma^{2} B^{2}}{\gamma m_{0} D} \rho_{\|}\left[\chi^{\prime}(s)+\left(\rho_{\|}+\Upsilon\right) \mu_{0} J^{\prime}(s)+\left.\mu_{0} J(s) \frac{\partial \Upsilon}{\partial s}\right|_{\theta, \varphi, t}\right] \\
& -\frac{e \sigma^{2} B^{2}}{\gamma m_{0} D} \rho_{\|}\left(\rho_{\|}+\Upsilon\right) \frac{\partial\left(\sigma B_{s}\right)}{\partial \theta} \\
& +\frac{\sigma B_{s}}{D}\left[\frac{\partial \Phi}{\partial \theta}+\frac{1}{\gamma}\left(\frac{\mu}{e}+\frac{e B}{m_{0}} \sigma \tau \rho_{\|}^{2}\right) \frac{\partial B}{\partial \theta}-\frac{e \sigma^{2} B^{2}}{\gamma m_{0}} \rho_{\|} \frac{\partial \Upsilon}{\partial \theta}\right],
\end{aligned}
$$




$$
\begin{aligned}
\dot{\rho}_{\|}= & -\left.\frac{\partial \Upsilon}{\partial t}\right|_{s, \theta, \varphi} \\
& -\frac{1}{D}\left[\psi^{\prime}(s)+\left(\rho_{\|}+\Upsilon\right) \mu_{0} I^{\prime}(s)+\left.\mu_{0} I(s) \frac{\partial \Upsilon}{\partial s}\right|_{\theta, \varphi, t}\right] \\
& \times\left[\left.\frac{\partial \Phi}{\partial \theta}\right|_{s, \varphi, t}+\frac{1}{\gamma}\left(\frac{\mu}{e}+\sigma \tau \frac{e B}{m_{0}} \rho_{\|}^{2}\right) \frac{\partial B}{\partial \theta}\right] \\
& -\frac{1}{D}\left[\chi^{\prime}(s)+\left(\rho_{\|}+\Upsilon\right) \mu_{0} J^{\prime}(s)+\left.\mu_{0} J(s) \frac{\partial \Upsilon}{\partial s}\right|_{\theta, \varphi, t}\right] \\
& \times\left[\left.\frac{\partial \Phi}{\partial \varphi}\right|_{s, \theta, t}+\frac{1}{\gamma}\left(\frac{\mu}{e}+\sigma \tau \frac{e B}{m_{0}} \rho_{\|}^{2}\right) \frac{\partial B}{\partial \varphi}\right] \\
& +\frac{\mu_{0}}{D}\left[\left.I(s) \frac{\partial \Upsilon}{\partial \theta}\right|_{s, \varphi, t}+\left.J(s) \frac{\partial \Upsilon}{\partial \varphi}\right|_{s, \theta, t}\right] \\
& \times\left[\left.\frac{\partial \Phi}{\partial s}\right|_{\theta, \varphi, t}+\frac{1}{\gamma}\left(\frac{\mu}{e}+\sigma \tau \frac{e B}{m_{0}} \rho_{\|}^{2}\right) \frac{\partial B}{\partial s}+\left.\frac{e \sigma B^{2}}{\gamma m_{0}} \rho_{\|} \frac{\partial \sigma}{\partial s}\right|_{B}\right] \\
& -\frac{1}{D}\left[\left(\rho_{\|}+\Upsilon\right) \frac{\partial\left(\sigma B_{s}\right)}{\partial \varphi}+\sigma B_{s} \frac{\partial \Upsilon}{\partial \varphi}\right] \\
& \times\left[\frac{\partial \Phi}{\partial \theta}+\frac{1}{\gamma}\left(\frac{\mu}{e}+\frac{e B}{m_{0}} \sigma \rho_{\|}^{2}\right) \frac{\partial B}{\partial \theta}\right] \\
& +\frac{1}{D}\left[\left(\rho_{\|}+\Upsilon\right) \frac{\partial\left(\sigma B_{s}\right)}{\partial \theta}+\sigma B_{s} \frac{\partial \Upsilon}{\partial \theta}\right] \\
& {\left[\frac{\partial \Phi}{\partial \varphi}+\frac{1}{\gamma}\left(\frac{\mu}{e}+\frac{e B}{m_{0}} \sigma \rho_{\|}^{2}\right) \frac{\partial B}{\partial \varphi}\right] } \\
& {[4 \mathrm{~d}) } \\
&
\end{aligned}
$$

with

$$
\begin{aligned}
D= & \psi^{\prime}(s) J(s)-\chi^{\prime}(s) I(s) \\
& +\left(\rho_{\|}+\Upsilon\right)\left[J(s) I^{\prime}(s)-I(s) J^{\prime}(s)+I(s) \frac{\partial\left(\sigma B_{s}\right)}{\partial \theta}\right. \\
& \left.+J(s) \frac{\partial\left(\sigma B_{s}\right)}{\partial \varphi}\right] .
\end{aligned}
$$

The relativistic correction can be written $\gamma=\sqrt{1+2 \mu B /\left(m_{0} c^{2}\right)+\left(m v_{\|}\right)^{2} /\left(m_{0}^{2} c^{2}\right)}, \Upsilon$ is the parallel vector potential perturbation, and $\tau=1+\left(\mu_{0} / B\right) \partial p_{\perp} /\left.\partial B\right|_{s}$ the mirror stability parameter as introduced in Ref. [17]. $\psi$ and $\chi$ are the poloidal and toroidal magnetic fluxes, $I$ and $J$ the poloidal and toroidal current fluxes. The terms containing $\sigma B_{s}$ assure the canonical properties, and are neglected in the standard formulation. The relativistic corrections are only important for the evolution of highly energetic electrons in magnetic confinement fusion plasmas. It is set to unity throughout this article. For more details, see Ref. [10].

\subsubsection{Interaction operators}

To advance the evolution of the distribution function, interactions with the background ions and electrons as well as the RF wave field have to be implemented. All interactions are described using Monte Carlo methods. Interactions with the background ions and electrons are modeled through Coulomb collision operators, in both pitch angle and energy. They are applied at every time step. The action of the wave field on the distribution function is included using kicks in velocity space (i.e. pitch angle and energy) every time a particle crosses the Doppler-shifted resonant layer [18].

Coulomb collisions. The defining parameters for Coulomb Monte Carlo operators are the slowing down $\tau_{s}$ and the deflection times $\tau_{d}$, describing characteristic time scales for energy and pitch angle collisions respectively. They are derived e.g. by Stix [19] and can be written [20]

$$
\begin{aligned}
\tau_{s}^{p} & =\frac{v_{t h, p}^{2} v}{\left(1+m / m_{p}\right) A_{D}^{p} \Psi(x)}, \\
\tau_{d}^{p} & =\frac{v^{3}}{A_{D}^{p}[\Phi(x)-\Psi(x)]},
\end{aligned}
$$

where we have defined $A_{D}^{p}=n_{p} q^{2} q_{p}^{2} \ln \Lambda / 2 \pi \epsilon_{0}^{2} m^{2}, x=v / v_{t h, p}$, $\Phi(x)=\operatorname{erf}(x) \equiv 2 / \sqrt{\pi} \int_{0}^{x} e^{-y^{2}} \mathrm{~d} y, \Psi(x)=\left(\Phi(x)-x \Phi^{\prime}(x)\right) / 2 x^{2}$ and $\ln \Lambda$ is the Coulomb logarithm. Super- or subscript $p$ stands for the background species. With these time scales we can construct the Monte Carlo Coulomb collision operators in energy and pitch angle. They are written in terms of collision frequencies $v_{d, s}=\sum_{p} v_{d, s}^{p}=$ $\sum_{p} 1 / \tau_{d, s}^{p}$, and applied at every time step using [21]

$$
\begin{aligned}
\lambda_{n+1}= & \lambda_{n}\left(1-v_{d} \tau\right)+\mathcal{R} \sqrt{\left(1-\lambda_{n}^{2}\right) v_{d} \tau}, \\
E_{n+1}= & E_{n}-\left(2 v_{s} \tau\right)\left[E_{n}-\left(\frac{3}{2}+\frac{E_{n}}{v_{s}} \frac{\mathrm{d} v_{s}}{\mathrm{~d} E}\right) T_{p}\right] \\
& +2 \mathcal{R} \sqrt{T_{p} E_{n} v_{s} \tau} .
\end{aligned}
$$

We write $\lambda \equiv v_{\|} / v$ for the pitch angle, $\tau$ for the numerical time step, $T_{p}$ for the background (thermal) temperature and $n$ labels the time steps. $\mathcal{R}$ are random numbers with zero mean value and unitary variance.

ICRH operators. To model the effect of an RF field on the particles' motion, Monte Carlo operators acting in velocity space are implemented. Following Kaufman's derivation [22], one can construct the Monte Carlo operator providing random kicks to the perpendicular velocity $[23,18]$

$\Delta v_{\perp}=\frac{\left\langle\Delta v_{\perp}^{2}\right\rangle}{4 v_{\perp}}+\mathcal{R} \sqrt{2\left\langle\Delta v_{\perp}^{2}\right\rangle}$,

with

$\sqrt{\left\langle\Delta v_{\perp}^{2}\right\rangle}=\tau \frac{q}{m}\left|E^{+} J_{n-1}+E^{-} J_{n+1}\right|$,

where $J_{n}$ denotes the Bessel function and takes as an argument $k_{\perp} v_{\perp} / \Omega . \Omega$ is the local cyclotron frequency and, as before, $\mathcal{R}$ is a random number with zero mean value and unitary variance. The average $\langle\cdot\rangle$ is over the random phase of the RF field with respect to the particle gyro motion. Introducing the phase $v(t) \equiv \int^{t}(\omega-$ $\left.k_{\|} v_{\|}-n \Omega\right) \mathrm{d} t^{\prime}$, the interaction time $\tau$ corresponds to the phase integral $[24,25]$

$\tau=\int^{t} \mathrm{~d} t^{\prime} e^{i v}$

We can expand the phase around the resonance (when $\dot{v}=\omega-$ $k_{\|} v_{\|}-n \Omega=0$ ) and write

$\left.\tau \approx \int_{-\infty}^{\infty} \mathrm{d} t \exp i\left(v+\frac{1}{2} \ddot{v} t^{2}+\frac{1}{6} \dddot{v} t^{3}\right)\right|_{\dot{\nu}=0}$,

where a dotted variable is a derivative with respect to time. In most cases, the expansion to the second order is sufficient, yielding

$\tau_{1}=\sqrt{2 \pi /|\ddot{v}|}$.

However, when $\ddot{v} \rightarrow 0$, one has to retain the third order term in (10), and one obtains [24]

$\tau_{2}=2 \pi(2 / \dddot{v})^{1 / 3} \operatorname{Ai}\left(-\ddot{v}^{2} /\left(2^{2 / 3} \dddot{v}^{4 / 3}\right)\right)$, 
where $\mathrm{Ai}$ is the Airy function. $\tau_{1}$ is used as default interaction time, but $\tau_{2}$ has to be used when $\ddot{v} \rightarrow 0$, in which case $\tau_{1} \rightarrow \infty$. More precisely, one can show that $\left(\tau_{2} / \tau_{1}\right)^{2}=1$ if $\dddot{v}^{2}=69.65 \ddot{\ddot{v}}^{3}$, so that we use $\tau_{2}$ if $\dddot{v}^{2}>69.65 \ddot{v}^{3}$. Additionally, for the kicks in parallel velocity, one invokes the conservation of total energy $\mathrm{d} E=\hbar \omega$ and toroidal momentum $\mathrm{d} P_{\varphi}=\hbar n_{\varphi}$, giving $\mathrm{d} P_{\varphi}=n_{\varphi} / \omega \mathrm{d} E$ [18]. We can locally express this through the Kennel-Engelmann constraint [26]

$v_{\perp}^{2}+\left(v_{\|}-\frac{\omega}{k_{\|}}\right)^{2}=$ const.

Together with the condition that at the resonance (at which this operator is only applied), $v_{\|}=(\omega-n \Omega) / k_{\|}$, we can write the Monte Carlo operator for the parallel velocity

$\Delta v_{\|}=\frac{k_{\|}}{n \Omega} v_{\perp} \Delta v_{\perp}$.

Including the change in parallel velocity $\Delta v_{\|}$is important when crossing the Doppler-shifted resonance, since it corresponds not only to a change in particle energy but also in the toroidal angular momentum $P_{\varphi}$, thus including the RF induced particle pinch. Indeed, the shift of the radial banana orbit displacement is proportional to the change in parallel velocity through [27]

$\Delta r \approx \frac{m}{q B_{\theta}} \Delta v_{\varphi} \approx \frac{m}{q B_{\theta}} \Delta v_{\|}$

as described e.g. in Refs. [28] and [29]. Eqs. (8) and (14) are the operators implemented in VENUS. They are applied every time a particle crosses the Doppler shifted resonance $\omega=k_{\|} v_{\|}+n \Omega$. As stated earlier, only fundamental ICRH $(n=1)$ is modeled here because LEMan is restricted to zeroth order terms in Larmor radius, and thus higher harmonic resonances cannot be dealt with.

\subsubsection{Wave numbers}

In the kicks in velocity space, operators (8) and (14), a parallel and a perpendicular wave number appears. For one given wave field, one value for $k_{\|}$and $k_{\perp}$ as a function of real space has to be passed from LEMan to VENUS. In LEMan, many modes are excited even for one given toroidal mode, and it is not evident to define one global wave vector to be used in the Monte Carlo operators in VENUS. Often, the simple $k_{\|}=n_{\varphi} / R$ approximation together with the local dispersion relation for the fast wave are used. Here, we propose a different approach, using the total scalar potential $\Phi$ computed in LEMan. The details of the computation are given in Appendix A, and we state here only the final form of the parallel and perpendicular wave numbers.

$$
\begin{aligned}
\left|k_{\|}\right|=\mid & \frac{1}{\Phi B \sqrt{g}}\left(\psi^{\prime} \frac{\partial \Phi}{\partial \theta}+\chi^{\prime} \frac{\partial \Phi}{\partial \varphi}\right) \mid, \\
\left|k_{\perp}\right|^{2}= & \left|g^{S S}\left(\frac{1}{\Phi} \frac{\partial \Phi}{\partial s}\right)\right|^{2} \\
& +\left|\frac{1}{g^{S S}}\left[\frac{1}{\Phi \sigma B \sqrt{g}}\left(\mu_{0} J \frac{\partial \Phi}{\partial \varphi}+\mu_{0} I \frac{\partial \Phi}{\partial \theta}\right)\right]\right|^{2} .
\end{aligned}
$$

$\sqrt{g}$ is the Jacobian, $g^{s s}$ the first metric element, the prime denote the derivative with respect to the radial variable $s$, and all other notations are as introduced earlier. It is important to note that Eqs. (16) and (17) are the values passed to VENUS for the Monte Carlo operators, and are not used in the wave computations in LEMan. Also, the operators (8) and (14) have been derived for the wave-particle interaction with one given wave, and for the notion of one single wave vector to make sense, we must assume that no strong reflection of the injected wave is occurring.

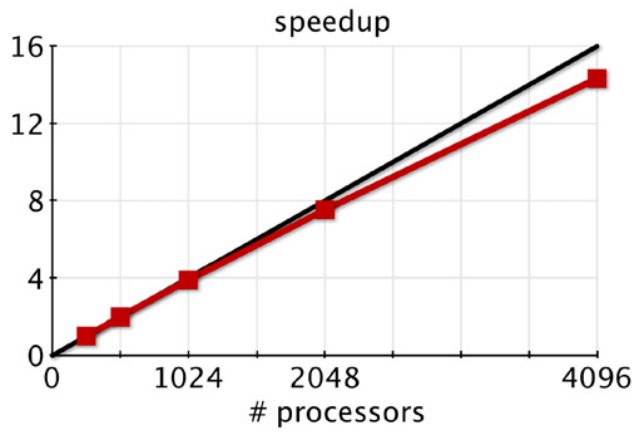

Fig. 3. Speedup of the VENUS code from 256 to 4096 processors (strong scaling) The straight line shows perfect scaling.

\subsubsection{Scaling}

Advancing the distribution function is the most time consuming part of SCENIC. Indeed, a two-dimensional equilibrium takes a few seconds on one single processor for VMEC. In an axisymmetric geometry and for one toroidal mode number, LEMan needs several minutes using two quadcore compute nodes to add the wave field information for one toroidal mode number in the axisymmetric case. The code uses the Burn At Both Ends (BABE) scheme on two compute nodes (using MPI) and is additionally OMP parallelised and can thus run even faster on machines having more cores per node. In contrast, VENUS requires of the order of tens of thousands of CPU hours for a converged solution (multiple iterations included), depending of course on the number of particles needed. In a typical run described in Section 4, four million markers were used, and a total converged simulation took about 30000 CPU hours. It is therefore important that VENUS scales well on high performance computers up to thousands of processors. The markers within VENUS do not interact with each other and thus the code is trivially parallelised (using MPI) by simply distributing the total number of markers among the processors. However, the simulation can of course not be done completely without communication among the processors, and the scaling is therefore not perfect. In the beginning, the equilibrium and wave field have to be read by all the processors. During the simulation, diagnostics are run at constant time intervals. These diagnostics involve communication among the processors, slowing down the simulation by a few percent for the benefit of diagnostics information. At the end of every VENUS simulation, the self-consistency modules (described in the next section) need the information of all the markers for integrating the distribution function and re-creating new inputs for VMEC and LEMan. Here, all information needed is given to the master processor and treated by the latter only. Again, the parts not parallelised use a few minutes of wall clock time compared with the tens of hours of the bulk of the simulation. Fig. 3 shows the speedup of parallelisation over thousands of processors and proves very satisfactory strong scaling.

\subsection{Self-consistency modules}

The evolution of the distribution function is simulated in VENUS. For the iteration between equilibrium, wave field and distribution function to be possible, outputs have to be generated from VENUS serving as inputs for a new iteration (red arrows in Fig. 1). One also has to be careful when taking the output of VENUS, since Monte Carlo simulations can be noisy. With the fast particle distribution in phase space provided by VENUS, the distribution function and its moments, such as density $n^{h}(s, \theta)$, perpendicular and parallel pressure $p_{\perp}^{h}(s, \theta), p_{\|}^{h}(s, \theta)$ and trapped and passing current $j_{t}(s, \theta), j_{p}(s, \theta)$, are known at the end of every iteration. These quantities will be used to compute the new inputs for the next iteration. In this way, the potentially noisy Monte 
Carlo distribution function is not directly used for the iterations, but rather the integrated moments. For the iterated scheme, only radial profiles are needed, and we can apply additional smoothing algorithms on the obtained profiles before using them as new inputs. The background electron and ion profiles (density and temperature) are considered static, i.e. constant during the simulation. Only the simulated minority is dynamically changing. But out of this minority population, especially for low to moderate heating power scenarios, only a certain portion is effectively heated to higher energies, depending on background profiles, heating location, type of minority, etc. It is then interesting to divide the minority population into a thermal and a hot species, and compute the moments of the distribution function for each of these two separately. Then, the thermal minority species can be treated as second thermal species in the dielectric tensor, and the thermal minority pressure added to the background pressure in the equilibrium. With this, only the energetic minority population is treated to be hot and bi-Maxwellian. We note that depending on the injected RF power, an important part of the minority species will become energetic, and thus a $\delta$ f scheme would not be appropriate anymore, since the condition $\delta \mathrm{f} \ll \mathrm{f}$ would not be satisfied. This is why we chose a full-f description in VENUS. In order to make the division into hot and thermal species automatic in the statistics section of VENUS, one needs a criterion of when a particular particle is to be considered hot or thermal. The knowledge of the background profiles is of help, since the thermal part of the minority population is expected to have a similar temperature as the background. This suggests a criterion based on the background temperature profile. In VENUS, a fairly simple procedure is implemented, which takes as limiting energy $E_{c}$ for any particle a multiple of the electron temperature at the radial position of the particle:

$$
\begin{aligned}
& E_{c}(s)=x T_{e}(s) \\
& \text { if } E_{p}<E_{c}(s) \Rightarrow \text { thermal } \\
& \text { else } \Rightarrow \text { hot, }
\end{aligned}
$$

with $E_{p}$ the particle's energy and $x$ a coefficient which will be explored in Section 3.4 and Figs. 8 and 9. This test is applied to every particle and the computed moments are then $n_{t h}^{h}, n^{h}, p_{t h}^{h}$, $p_{\perp}^{h}$ and $p_{\|}^{h}$, where the subscript th denotes quantities which contain only contributions from minority particles with a lower energy than $E_{c}(s)$. Quantities without that subscript are computed with the particles having an energy $E_{p}>E_{c}$ only. Note that the induced currents, trapped $j_{t}$ and passing $j_{p}$, have to be taken from the complete distribution, since the total toroidal current has to be passed to VMEC.

As described in Section 2.1 and Ref. [6], the inputs to the equilibrium code VMEC are the background and hot particle pressures and the total toroidal current. With our splitting mechanism, the background pressure will also contain the pressure due to the thermal part of the minority population, $p_{t h}^{h}$. Furthermore, the RF induced current (ICCD) can directly be added to the ohmic current for the equilibrium computations.

LEMan assesses the constant background density and temperature profiles once at the beginning of the simulation. In addition it reads at every iteration the density and temperature profiles of the thermal minority (coming directly from the thermal part of the distribution function in VENUS) and for the hot bi-Maxwellian population the anisotropy $T_{\perp}(s) / T_{\|}(s)$, the density-like amplitude factor $\mathcal{N}(s)$ introduced in Section 2.1 and the profile of the hot parallel temperature $T_{\|}(s)$. All of these quantities depend directly or indirectly on the magnetic field strength and the moments of the hot particle distribution function. It is important to specify that these moments are integrated directly at the end of every VENUS

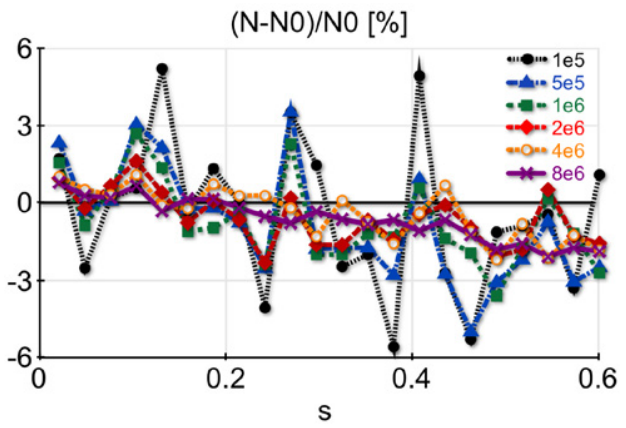

Fig. 4. Scan using different number of particles in VENUS. The density factor $\mathcal{N}(s)$ has been recreated directly after initial loading and compared to the input value $\mathcal{N}_{0}(s)$. The scan is performed from $10^{5}$ to $8 \times 10^{6}$ particles with a grid size of $72 \times 55$.

run. There are no approximations on the form of the distribution function itself. Only when computing the required radial profiles of derived quantities (such as $T_{\perp} / T_{\|}, \mathcal{N}$, and $T_{\|}$) are we using relations coming from the bi-Maxwellian model. The relevant relations are given in Appendix B, and further details can be found in Ref. [6].

\section{Code validation}

After the description of the different elements of SCENIC, we turn our attention to some elementary testing. The Coulomb operators are standard and widely used, and we will simply show that they do indeed cause the distribution function to relax to an isotropic Maxwellian of the same temperature as the background. The RF Monte Carlo operators will be checked in two ways. They have to reproduce the power deposition pattern computed by LEMan, provided that the power is sufficiently low such that the heating does not have any effect on the dielectric tensor. Secondly, the RF induced particle pinch $[28,29]$ has to be observed in the simulations. Finally, we will check the splitting mechanism described in Section 2.4. In the subsequent plots, we will not use the radial Boozer coordinate $s$ as a radial variable, but the more intuitive normalised radius $r / a$. Here, $a$ is the minor radius, and the coordinates are approximatively linked through $s \sim(r / a)^{2}$.

\subsection{Number of particles}

The number of particles required in VENUS depends to a large extent on the chosen simulation. Depending on the heating scenario, pressure and density profiles (and thus also $T_{\perp} / T_{\|}$and all other derived quantities) can show strongly localised maxima in both radial and poloidal direction. It is thus helpful to have a rather high number of grid points, up to $96 \times 75$ (radial $\times$ poloidal). Additionally, when iterating between the codes, smooth profiles are needed at every iteration. This asks for a high number of particles. However, when studying a low power scenario, or a case where broad maxima can be expected in the profiles, fewer grid points can be used, decreasing the required number of particles. Fig. 4 shows a scan over the number of particles using a $72 \times 55$ grid. For this check, we loaded the particles and directly created the VENUS outputs, without advancing the orbits in time. The resulting outputs should be equal to the inputs. Fig. 4 then gives the relative difference between input and output of the density factor $\mathcal{N}(s)$ as an example, and we do not consider the values of the radial variable $s>0.6$ for more clarity. The relative error diminishes for higher particle number, but the curves of four and eight million particles are rather close to each other and not much different from two million, suggesting a certain saturation. The Coulomb collision tests in Fig. 5 have been performed with one million particles on a $36 \times 55$ grid, whereas the results shown in Section 4 


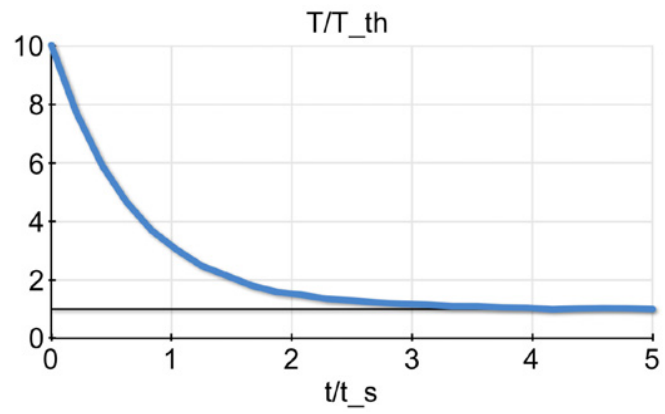

(a)

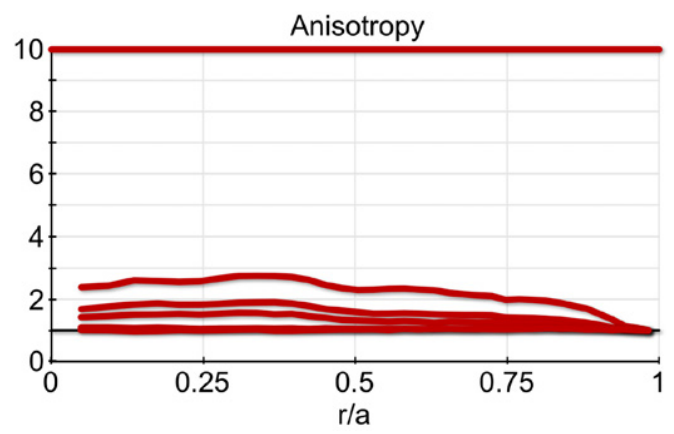

(b)

Fig. 5. Coulomb collisions have the effect that any minority distribution function will become isotropic and have the same temperature as the background species. Anisotropy denotes the factor $T_{\perp} / T_{\|}$. The simulations were conducted using one million particles on a $36 \times 55$ grid. (a) The Coulomb collision operators cool down an initially ten times warmer distribution to the background temperature. The black horizontal line denotes $T=T_{t h}$. (b) The pitch angle operator leads to an isotropic distribution. The figure shows radial profiles for $0,0.5,1,1.5,3$ and 4.5 deflection times. The black line indicates unity.

have been produced following four million particles on $72 \times 55$ grid.

\subsection{Coulomb Monte Carlo operators}

The Monte Carlo operators for the Coulomb collisions on the background ions and electrons are described in Eq. (7) and act on the pitch angle and energy. We can easily test those two operators considering a given background Maxwellian of constant density and temperature. For the slowing down, the collision operators have to cool down any initially hotter minority distribution to a Maxwellian of equal temperature within a few slowing down times. Fig. 5(a) shows such a test, where the minority species was initially ten times hotter than the background ions and electrons and converges to the same temperature within about four slowing down times.

The pitch angle scattering operator must produce the result that any initially anisotropic distribution ends up being isotropic. We can check this with an initial perpendicular temperature which is ten times higher than the parallel temperature, $T_{\perp}=10 T_{\|}$, $T_{\|}=T_{t h}$, and let the system evolve with the pitch angle scattering enabled. In Fig. 5(b) one can easily verify that the anisotropy does indeed tend to unity within a few deflection times. Note that the anisotropy is equalised first at the boundary, which is due to trapped particle losses on edge. Also, the deflection time depends strongly on the particle's energy, being shorter for lower energy. We used the deflection time $\tau_{d}$ corresponding to the initial energy distribution and ran the simulation for $4.5 \tau_{d}$.

\subsection{RF Monte Carlo operators}

The main goal of the quasilinear RF operators (8) and (14) is to ensure correct power deposition. As one of its outputs, LEMan

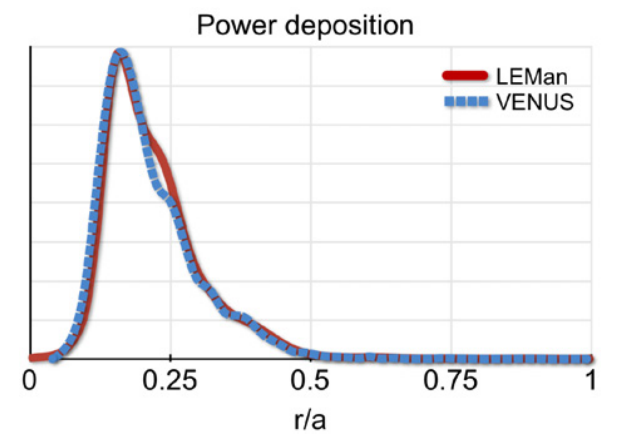

(a)

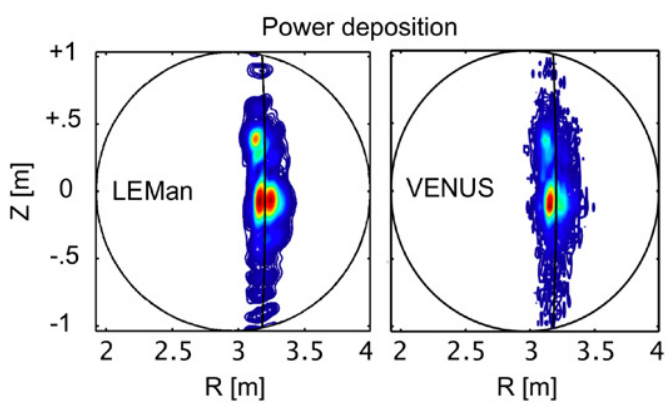

(b)

Fig. 6. The RF quasilinear operators reproduce accurately the power deposition of the RF wave. Here, the particles in VENUS were thermal with small orbit width, and also very low power was applied. With this, the exact same distribution is contained in LEMan and VENUS, such that a direct comparison was possible. (a) Normalised radial absorption profiles. The absolute values of the deposited power in LEMan and VENUS differs, since LEMan computes the power corresponding to an antenna current of $1 \mathrm{~A}$, whereas VENUS rescales the electric fields to obtain the wanted absorbed power for a given simulation. (b) $2 \mathrm{D}$ absorption. The maxima are located to the right and the left of the resonant layer due to a non-zero Doppler shift for a warm distribution on one hand, and, more importantly, a minimum along the resonant layer of the left handed polarized electric field $E^{+}$.

produces the power deposition as a radial profile (power density) and in two dimensions across a poloidal cross section. However, this power deposition is not used in VENUS, since the RF operators only need the wave field and the wave vectors as input from LEMan. We can therefore use the power deposition in LEMan and in VENUS as independent data and use it as a powerful check for the Monte Carlo operators. Fig. 6 shows the very satisfactory comparison between LEMan and VENUS using the normalised radial absorption profiles and the $2 \mathrm{D}$ absorption in the $R Z$-plane. For this comparison, we followed thermal minority ions, and applied only very little power, such that the distribution in VENUS was the same as in LEMan, and therefore conditions in LEMan and VENUS where as similar as possible.

Fig. 7 shows the evolution of the orbit of an initially passing particle which is being trapped and moving towards the resonance due to wave-particle interactions. Such a resonating particle is subject to the RF induced pinch, as described in Refs. [28] and [29] and Eq. (15). It arises due to a change in toroidal angular momentum $P_{\varphi}=m R v_{\|} B_{\varphi} / B+Z e \psi$ when interacting with the RF field. In VENUS, this effect is taken into account through the KennelEngelmann relation Eq. (13).

\subsection{Checking the splitting mechanism}

In Section 2.4 we described how the test particle distribution in VENUS can be mapped onto a sum of a Maxwellian and a bi-Maxwellian distribution describing the thermal and fast ions respectively. In particular, a critical energy is defined, below which particles are considered Maxwellian (thermal), and above which they are considered bi-Maxwellian and hot, as described 


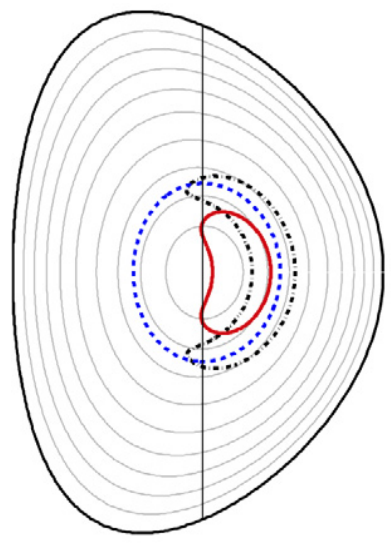

Fig. 7. Evidence of the RF induced particle pinch. An initially passing particle (blue, dashed) becomes trapped (black, dashed) and the turning points move towards the resonance (red, solid), yielding a potato-like orbit. The vertical black line represents the resonant layer. The orbit averaged radial position $(s$ in the model distribution function (1)) has moved towards the magnetic axis. (For interpretation of the references to color in this figure legend, the reader is referred to the web version of this article.)

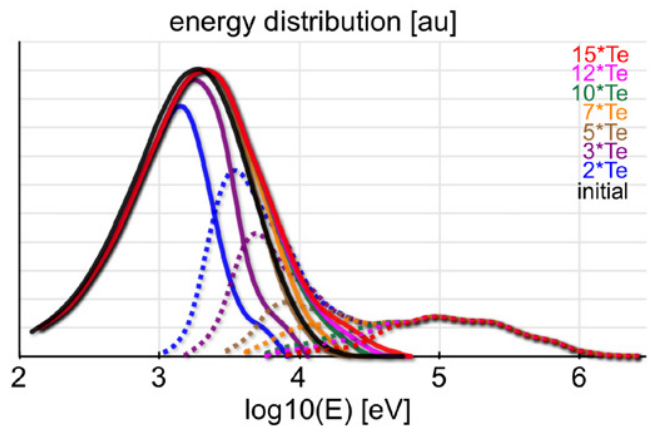

(a)

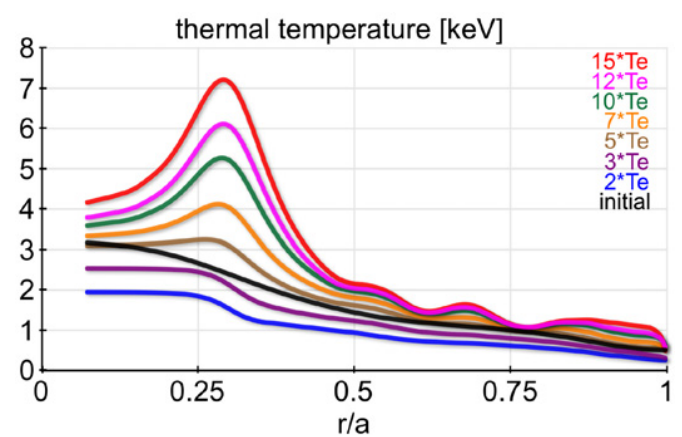

(b)

Fig. 8. Changing the critical energy for splitting the distribution function. Conditions (18) are used for the splitting and in the legends the value of the coefficient $x=E_{c} / T_{e}$ indicated. (a) Energy distribution $f(E) \sqrt{E}$ integrated over space and pitch angle. Thermal distribution in solid and tail in dotted lines. (b) The resulting thermal temperature profiles.

in Eq. (18). Fig. 8 shows a scan of the coefficient $x$ in Eq. (18), i.e. different multiples of the electron temperature as critical energies. We chose to plot the energy distribution in terms of $f(E) \sqrt{E}$, corresponding to the number of particles at a given energy (i.e. integrated over real space and pitch angle). The energy is represented in a logarithmic scale, such that the thermal part and the tail are visible as two distinct local maxima. The black curve shows the initial (thermal) distribution and profile (same temperature as background and isotropic).

In Fig. 8(a), one can see that a critical energy of two or three times the electron temperature is too small. Too large a fraction

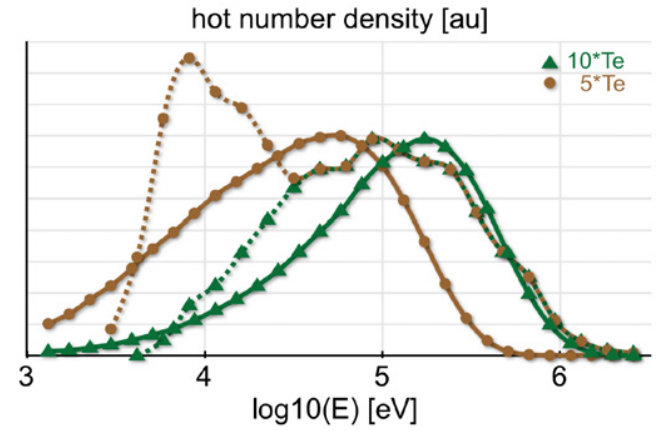

Fig. 9. VENUS output of the tail (dotted lines) compared to resulting analytical biMaxwellian. Clearly the factor of ten (green, triangles) is closer than the factor of five (brown, circles). (For interpretation of the references to color in this figure legend, the reader is referred to the web version of this article.)

of the thermal minority component (solid line) is assigned to the hot part, which is peaked far away from the tail (which is around $100 \mathrm{keV}$ in that case). In that plot, a factor of five is the best choice for representing the thermal minority part. This is confirmed by the second plot, Fig. 8(b), where the temperature profile for the factor of five is closest to the electron temperature (black). However, the main goal of this splitting is to be able to represent the tail as accurately as possible with the bi-Maxwellian model. As one can see from Fig. 8(a), the tail is peaked inside the initial thermal curve when using a factor of five, and representing the tail (outside the black curve) will be difficult with this splitting. This is what is shown in Fig. 9: The line representing the case of $E_{c}=5 T_{e}$ (brown, circles) incorporates a significant number of thermal particles (dotted line), and the analytical modeling for VMEC and LEMan (continuous lines) is further away from the form of the VENUS tail than the case $E_{c}=10 T_{e}$ (green, triangles). It becomes clear that choosing the value of $E_{c}$ is a trade-off between keeping the thermal part at the same temperature as the background and trying to get the analytical model to agree as much as possible in the tail. It is important to remember here that the particle distribution in VENUS is not modified with the splitting and is re-loaded at every iteration the way it was at the end of the previous iteration (i.e. non-bi-Maxwellian). Consequently, the exact choice of the critical energy is not as crucial as it seems, since the real minority distribution in VENUS is not directly affected. It is only the model distribution function applied to VMEC and LEMan which is directly influenced by the exact choice of $E_{c}$. Another note to make is that these checks were done for low power. For higher power, the tail will be more clearly separated from the bulk, and the choice for the critical energy is much more obvious.

\subsection{D calculations}

In this article, we focus primarily on (2D) tokamak equilibria due to the higher relevance to experiment. Nevertheless, in this section we show results obtained for a three-dimensional plasma to explicitly show the 3D capability of SCENIC. The geometry is defined by a 2-field period quasi-axisymmetric stellarator with zero toroidal current, based on a configuration discussed in Ref. [6], and scaled to a size similar to a typical JET equilibrium. The major radius is $R_{0}=2.91 \mathrm{~m}$, the magnetic field on axis $B_{0}=2.57 \mathrm{~T}$, and the volume averaged beta is $0.5 \%$. We consider a $1 \%$ helium3 minority in a deuterium plasma, and the wave frequency of $f=28 \mathrm{MHz}$, yielding $B_{c}=2.8 \mathrm{~T}$. This configuration is convenient because although it is fully three-dimensional, the magnetic field strength spectrum in Boozer coordinates is dominated by its axisymmetric components, such that the power deposition for ICRH can still be expected to lie along a well defined line of resonance at each given toroidal angle. We choose a low power scenario of 

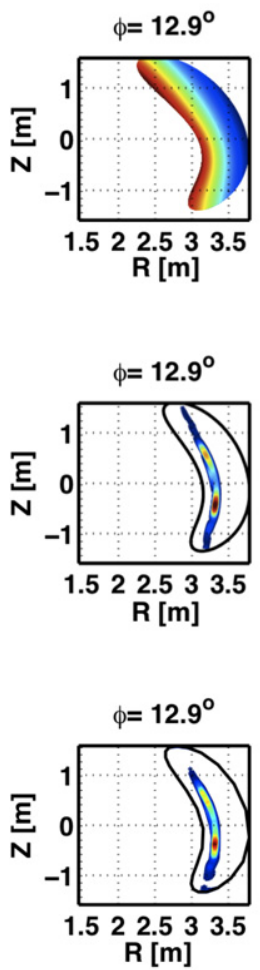
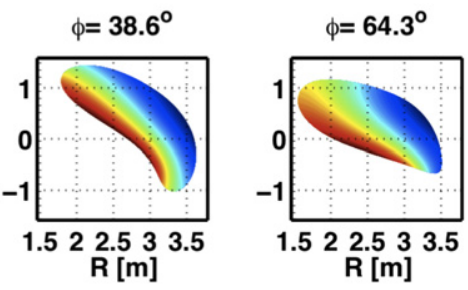

(a)
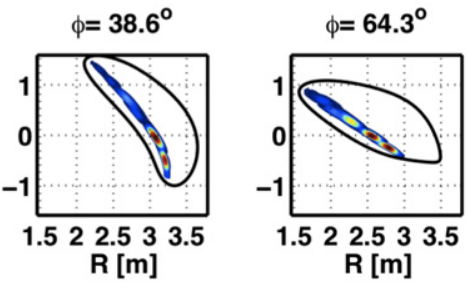

(b)
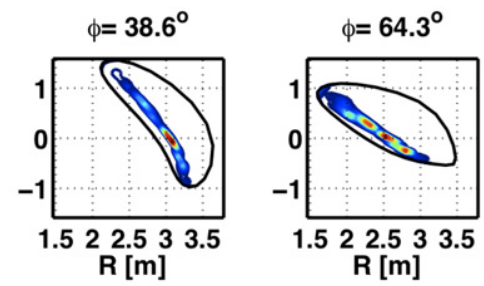
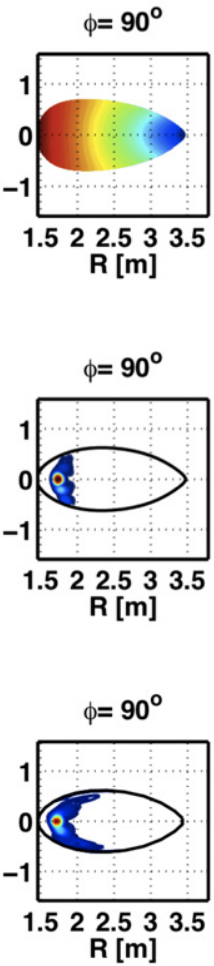

(c)

Fig. 10. 3D equilibrium, power deposition in the wave code LEMan and the Monte Carlo operators in VENUS for a quasi-axisymmetric configuration, shown at different toroidal angles. The figures demonstrate the 3D capability of SCENIC and its components. (a) Magnetic field strength from VMEC for a quasi-axisymmetric 2-field period stellarator. (b) Power deposition in LEMan. (c) Power deposition in the VENUS Monte Carlo operators.

$3 \mathrm{~kW}$, allowing for a direct comparison of the power deposition in the wave and the particle codes without the additional effect of Doppler broadening of the resonant layer. Fig. 10(a) shows the magnetic field strength and Figs. 10(b) and 10(c) the power deposition in both LEMan and the VENUS Monte Carlo operators at different toroidal angles. Figs. 10(b) and 10(c) provide an excellent demonstration of the functionality of SCENIC in 3D systems. In particular, as also seen in the 2D case of Fig. 6, the Monte Carlo operators deposit power to the single particles in correspondence with the power deposited by the RF electromagnetic wave, even over the toroidal angle. For such configurations, the power deposition is located along a given resonant layer. However, due to the important change of plasma shape along the toroidal direction, the orientation of power deposition reveals strong dependence on the toroidal angle. We conclude that SCENIC is indeed capable of simulating three-dimensional configurations.

\section{Iterated simulations}

After detailed description and individual checking, we will now show that the SCENIC package is capable of iterating between the codes and finding converged solutions. We will for this section turn to a JET-like equilibrium described in Appendix $C$ with $3 \mathrm{MW}$ of coupled ion cyclotron resonant heating. We use as a test case a $1 \%$ helium-3 minority in a deuterium plasma, heated on the high field side of the magnetic axis. With such a scenario, the fundamental $\mathrm{He}^{3}$ is the only resonance in the plasma. In order to check if the iterative method achieves convergence and to determine when the simulation can be stopped, a criterion easy to implement and observe has to be identified. Preferably it would be a global quantity, which does not depend on any position in phase space but which shows when a steady-state has been achieved. A steady-state is reached when the deposited power by the RF field is balanced by the power loss of the minority species to the back-

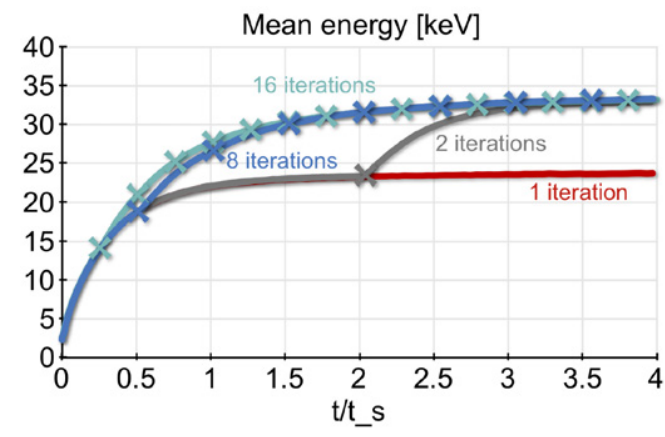

Fig. 11. Convergence of the iterated package SCENIC. Comparison of simulations without iterating (one iteration) and several iterations shows that even if they both reach equilibrium, the final results are different.

ground species. When that happens, the total energy content of the minority species will remain constant. Therefore, a relevant quantity to observe is the total energy content of the minority species, or, equivalently, the mean energy

$$
\langle E\rangle=\frac{1}{N} \int_{V} \frac{1}{2} m v^{2} f \mathrm{~d} V,
$$

where $V$ is the total phase space volume, $\mathrm{d} V=\mathrm{d}^{3} x \mathrm{~d}^{3} v$, and $N=\int_{V} f \mathrm{~d} V$. Fig. 11 shows an example of the evolution of the mean energy as a function of time for a $3 \mathrm{MW}$ simulation. Crosses denote starting of a new iteration and the time is normalised to the electron slowing-down time. In all cases, the system reaches a steady-state after a few (electron) slowing-down times. However, Fig. 11 shows clearly that iterations are indeed necessary, since the final results differ. The difference is obvious in Fig. 11, and it is also visible in the integrated moments of the distribution function. Confirming the difference in energy content of the minor- 


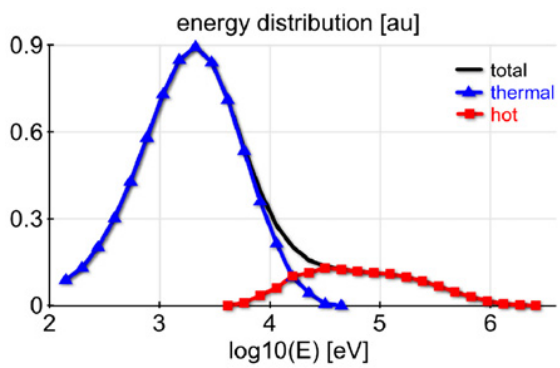

(a)

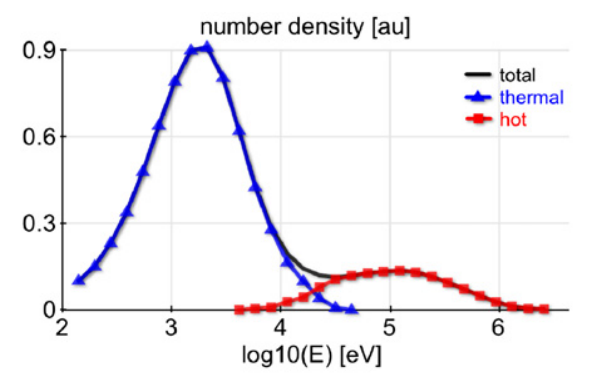

(b)

Fig. 12. Comparisons of the energy distribution $f(E) \sqrt{E}$. The tail of the iterated simulations is peaked just above $100 \mathrm{keV}$, whereas the tail in the simple simulation with one iteration has its maximum well below this mark. (a) 1 iteration. (b) 2 16 iterations. (For interpretation of the references to color in this figure legend, the reader is referred to the web version of this article.)

ity species, Fig. 12 gives the difference in the energy distribution $f(E) \sqrt{E}$, i.e. the number of particles at a given energy. For the chosen scenario, two iterations are enough, since convergence is attained against simulations with more and shorter iterations. One requires more iterations for higher power simulations. The splitting parameter is $x=10$ in both plots, and clearly the simulation with two and more iterations shows a high energy tail (red, squares) peaked at higher energy than the simple simulation with just one iteration. The difference in power deposition can be seen in Fig. 13. Whereas the deposition is rather narrow along the resonant layer for one iteration (Fig. 13(a)), it becomes quite wide at the end of the converged simulations using several iterations (Fig. 13(b)). Here, the difference between one iteration and several iterations may be attributed to the Doppler broadening of the resonance at higher energies and a change in electric field polarisation during the iterated simulations. Also, the radial deposited power density in Fig. 13(c) reflects the difference in local maxima of Fig. 13, and this plot will give an explanation for the difference in hot particle density, Fig. 14(b).

Fig. 14 illustrates the differences occurring in the profiles of pressure and density. For the cases using multiple iterations, the pressure is generally higher than for the case with one iteration, confirming the higher energy content proportional to the surface under the curves of Fig. 12. The one iteration density plot clearly shows local maxima, separated by a regular distance. It is important to remember that for these plots, only the resonant particles with energies higher than $E_{c}$ are considered. A direct relation between the hot particle density (Fig. 14(b)) and the radial power deposition (Fig. 13(c)) must be expected, since particles are heated where the power is absorbed. The important heating around $r / a=0.25$ in the beginning of the simulation (during the complete simulation for one iteration) creates a main hot particle density peak. The changed density then changes (together with the action of enhanced anisotropy) the power deposition after the initial iteration, enhancing single pass absorption, resulting in the shown differences between one and multiple iterations. These plots thus demonstrate that an iterated scheme is necessary for

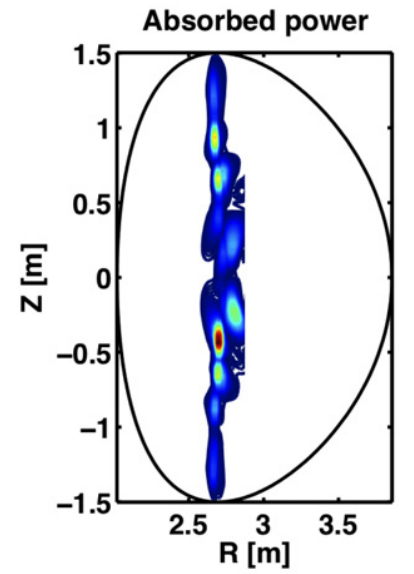

(a)

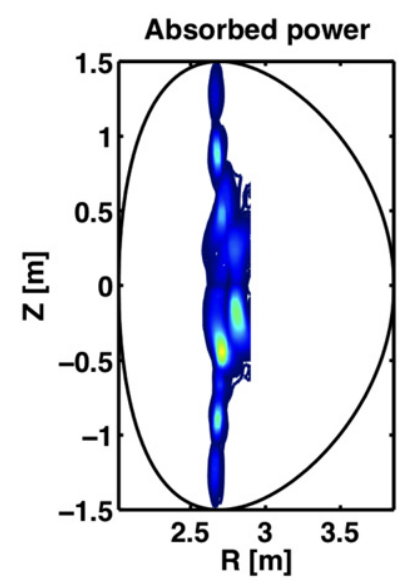

(b)

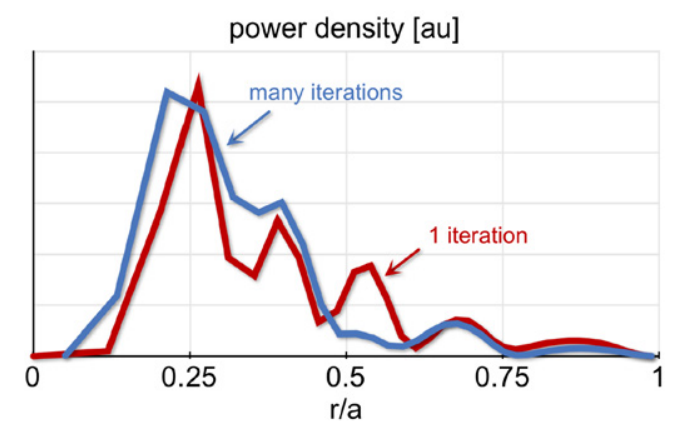

(c)

Fig. 13. Power deposition in VENUS. The power deposition becomes broader, and the single maxima along the resonance of (a) are less isolated in (b). The sharp boarder in (b) is due to the limited diagnostics grid size. (a) One iteration. (b) Last of 16 iterations. (c) Radial (poloidally averaged) deposited power density. Note that the grid points for the two curves are not at the same positions.

finding consistent solutions when performing ion cyclotron heating simulations.

When taking a closer look at the results presented, the relevance of the SCENIC modeling approach becomes apparent. We concentrate now on the converged multiple iteration results. At the end of the final iteration, we can take the zeroth, first and second moments of the distribution function in VENUS, yielding the density, current and pressure. Integrating over all variables except radius, one-dimensional profiles such as the already discussed pressure and density of Fig. 14 can be produced for physical studies. Fig. 15 shows the pressure and current profiles. The pressure shows an important difference between perpendicular (solid line) and parallel (dashed) pressure. This is intrinsically due to the 


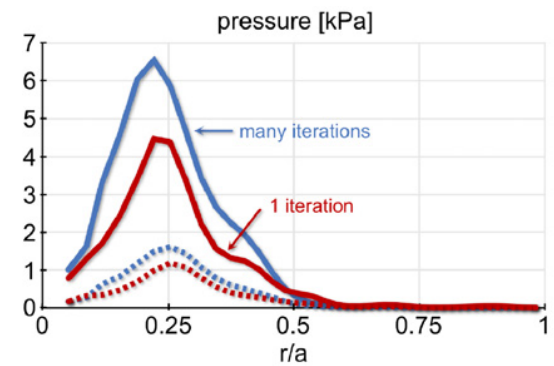

(a)

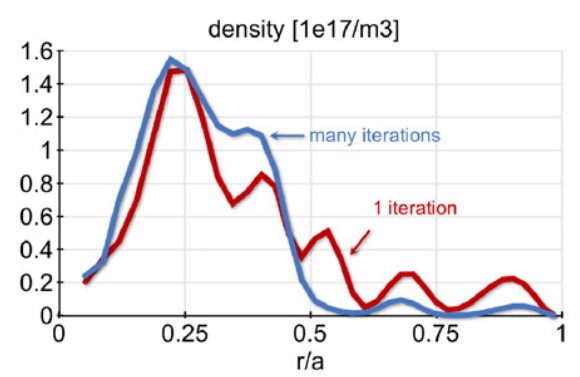

(b)

Fig. 14. Comparing integrated moments of the distribution function for pressure and density between one (red) and 2-16 (blue) iterations. Higher pressure is consistent with higher energy content in Fig. 11. The difference in density profiles (only the hot particle density is plotted) is directly linked to the difference in power deposition in Fig. 13. (a) Pressure, perpendicular (line) and parallel (dashed). (b) Particle density. (For interpretation of the references to color in this figure legend, the reader is referred to the web version of this article.)

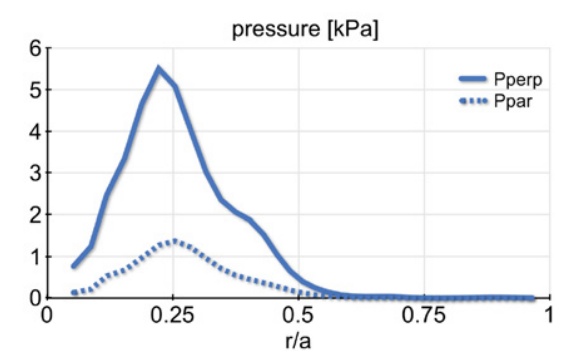

(a)

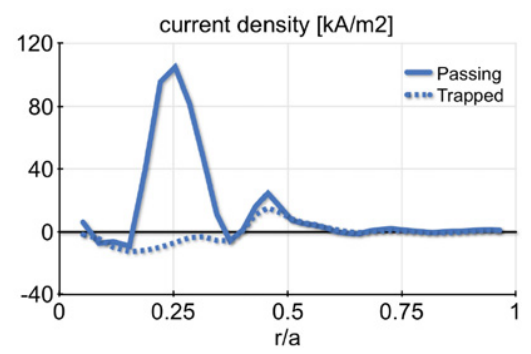

(b)

Fig. 15. Radial profiles of the hot particle pressure and current density. The difference between parallel and perpendicular pressure demonstrates the importance of including pressure anisotropy in the equilibrium and the dielectric tensor. SCENIC includes the RF induced current in the diagnostics of VENUS and the computation of the new equilibrium. (a) Hot minority pressure. $p_{\perp}$ solid, $p_{\|}$dashed. (b) Ion Cyclotron Current Drive (ICCD). Passing particles solid, trapped dashed.

mechanism of ICRH, since the heating is applied using the resonance with the perpendicular motion of the particle. Hence, the perpendicular pressure is much higher than the parallel pressure, and automatically the anisotropy $T_{\perp} / T_{\|}$is far from unity. In contrast to other models, our simulations are capable of including this anisotropy in all parts of the package and are therefore particu- larly well suited for ICRH simulations. Furthermore, it treats the $\mathrm{RF}$ induced current (Ion Cyclotron Current Drive (ICCD), Fig. 15(b)) self-consistently because it computes the various contributions to the current, including the RF induced particle pinch and finite orbit width effects, and then includes it in the equilibrium calculation. In the here shown low power scenario, a significant hot particle pressure anisotropy has developed (as shown in Fig. 15(a)), but the change to global measures of the equilibrium, like e.g. Shafranov shift or safety factor where small due to the low power employment. The goal of this first applications was not to push the simulations to their limits, but rather to prove the feasibility of this ambitious package, and high power as well as 3D geometry studies are left for future work. We conclude consequently that SCENIC represents an important improvement with respect to comparable code packages, showing good scaling on high performance computers and successful convergence of the iteration scheme. Iterations are important for obtaining meaningful results, as well as taking into account quantities such as pressure anisotropy and RF induced currents.

\section{Summary and conclusions}

We have described the development and first applications of the new numerical code package SCENIC, designed for Ion $\mathrm{Cy}-$ clotron Resonant Heating (ICRH). The code package is composed of a bi-Maxwellian anisotropic pressure version of the MHD equilibrium code VMEC, the full wave code LEMan and the particle-in-cell code VENUS. It is more general in many ways than any of the existing codes, in that it is capable of dealing with three-dimensional geometry including full shaping, pressure effects and pressure anisotropy. Using a separation of species into thermal background ions and electrons, thermal and hot minority ion species, different models are implemented for the equilibrium and dielectric tensor. Whereas a warm Maxwellian (isotropic) is applied to the background and the thermal minority species, the hot minority pressure in the equilibrium and the hot minority dielectric tensor of the wave code are treated anisotropic bi-Maxwellian. The evolution of the minority species' distribution function is computed in VENUS using Monte Carlo operators in pitch angle and energy space for both Coulomb collisions with the background and waveparticle interactions. Here, no approximation is made concerning the distribution function within the guiding centre model. The integrated moments from the distribution function can then be fed into the equilibrium and wave calculations, closing the loop and thus allowing for the search of a self-consistent solution. We have shown the consistency of the different modules and results using extensive tests of the code package. These tests included a JET-like equilibrium as well as a quasi-axisymmetric 2-field period stellarator [6], demonstrating the ability to treat both 2D and fully $3 \mathrm{D}$ geometries. Moreover, the scaling of the code on thousands of processors has been determined. Applying SCENIC to experimentally relevant JET-like configurations [1], we could establish the robustness of the iterative method and show that to compute a consistent solution, several iterations are essential. Finally, modeling pressure anisotropy and RF induced currents in SCENIC could be confirmed as an important feature for ICRH calculations.

\section{Acknowledgements}

This work was supported by part by the Swiss National Science Foundation. We thank S.P. Hirshman for the use of the VMEC code. Computations for this article have been performed on the HPCFF cluster at the Jülich Supercomputing Center (JSC), Germany, and on the Rosa XT-5 platform at the Swiss National Supercomputing Center in Manno, Switzerland. 


\section{Appendix A. Wave number computation}

A.1. Basis vectors

$\mathbf{e}^{s}=\nabla s$

$\mathbf{e}^{\theta}=\nabla \theta$

$\mathbf{e}^{\varphi}=\nabla \varphi$

$\mathbf{e}_{s}=\sqrt{g}(\nabla \theta \times \nabla \varphi)$,

$\mathbf{e}_{\theta}=\sqrt{g}(\nabla \varphi \times \nabla s)$,

$\mathbf{e}_{\varphi}=\sqrt{g}(\nabla s \times \nabla \theta)$,

with

$\sqrt{g}=\frac{1}{\nabla s \cdot(\nabla \theta \times \nabla \varphi)}$.

Magnetic field components in Boozer coordinates

$B^{S}=0$

$B^{\theta}=\psi^{\prime} / \sqrt{g}$,

$B^{\varphi}=\chi^{\prime} / \sqrt{g}$,

$B_{s}=B_{s}$,

$B_{\theta}=\mu_{0} J / \sigma$,

$B_{\varphi}=-\mu_{0} I / \sigma$,

with $I$ the poloidal current flux, $J$ the toroidal current flux, $\psi$ the poloidal and $\chi$ the toroidal flux functions. Prime denotes the derivative with respect to $s$. We will always compute the wave numbers using the scalar potential.

\section{A.2. Alternative basis}

For the computation of the wave vectors, let us define new basis vectors as

$\mathbf{e}^{s}=\nabla s$,

$\mathbf{e}^{b}=(\mathbf{b} \times \nabla s)$,

$\mathbf{e}^{\|}=\mathbf{b} \equiv \frac{\mathbf{B}}{B}$,

$\mathbf{e}_{s}=\sqrt{\mathcal{J}}\left(\mathbf{e}^{b} \times \mathbf{e}^{\|}\right)$,

$\mathbf{e}_{b}=\sqrt{\mathcal{J}}\left(\mathbf{e}^{\|} \times \mathbf{e}^{s}\right)$,

$\mathbf{e}_{\|}=\sqrt{\mathcal{J}}\left(\mathbf{e}^{s} \times \mathbf{e}^{b}\right)$.

The Jacobian is

$$
\begin{aligned}
\sqrt{\mathcal{J}} & =\frac{1}{\nabla s \cdot(\mathbf{b} \times \nabla s \times \mathbf{b})} \\
& =\frac{1}{\nabla s \cdot((\mathbf{b} \cdot \mathbf{b}) \nabla s-(\mathbf{b} \cdot \nabla s) \mathbf{b})}=\frac{1}{\nabla s \cdot \nabla s},
\end{aligned}
$$

which is as expected since $e^{s} \perp e^{b} \perp e^{\|}$and $e^{s} \cdot e^{s} \neq 1$. Now,

$$
\begin{aligned}
\mathbf{e}_{s} & =\sqrt{\mathcal{J}}\left(\mathbf{e}^{b} \times \mathbf{e}^{\|}\right) \\
& =\sqrt{\mathcal{J}}((\mathbf{b} \times \nabla s) \times \mathbf{b})=\sqrt{\mathcal{J}} \nabla s=\sqrt{\mathcal{J}} \mathbf{e}^{s}, \\
\mathbf{e}_{b} & =\sqrt{\mathcal{J}}\left(\mathbf{e}^{\|} \times \mathbf{e}^{s}\right)=\sqrt{\mathcal{J}}(\mathbf{b} \times \nabla s)=\sqrt{\mathcal{J}} \mathbf{e}^{b}, \\
\mathbf{e}_{\|} & =\sqrt{\mathcal{J}}\left(\mathbf{e}^{s} \times \mathbf{e}^{b}\right) \\
& =\sqrt{\mathcal{J}}(\nabla s \times(\mathbf{b} \times \nabla s))=\sqrt{\mathcal{J}}(\underbrace{\nabla s \cdot \nabla s}_{1 / \sqrt{\mathcal{J}}}) \mathbf{b}-(\underbrace{\nabla s \cdot \mathbf{b}}_{0}) \nabla s=\mathbf{e}^{\|} .
\end{aligned}
$$

A.3. Perpendicular wave number $k_{\perp}$

By definition of the coordinates,

$$
k_{\perp}^{2}=k_{s} k^{s}+k_{b} k^{b},
$$

with

$$
\begin{aligned}
k^{s}= & \frac{1}{\Phi} \mathbf{e}^{s} \cdot \nabla \Phi \\
= & \frac{1}{\Phi} \nabla s \cdot \nabla \Phi=\frac{1}{\Phi}\left(\frac{\partial \Phi}{\partial s} \nabla s+\frac{\partial \Phi}{\partial b} \nabla b+\frac{\partial \Phi}{\partial \|} \nabla \|\right) \\
= & \frac{1}{\Phi}(\nabla s \cdot \nabla s) \frac{\partial \Phi}{\partial s}=\frac{1}{\Phi \sqrt{\mathcal{J}}} \frac{\partial \Phi}{\partial s}, \\
k_{s}= & \frac{1}{\Phi} \mathbf{e}_{s} \cdot \nabla \Phi=\frac{1}{\Phi} \sqrt{\mathcal{J}} \mathbf{e}^{s} \cdot \nabla \Phi \\
= & \sqrt{\mathcal{J}} k^{s}=\frac{1}{\Phi} \frac{\partial \Phi}{\partial s} \\
\Rightarrow & k_{s} k^{s}=\frac{1}{\sqrt{\mathcal{J}}}\left(\frac{1}{\Phi} \frac{\partial \Phi}{\partial s}\right)^{2}, \\
k^{b}= & \frac{1}{\Phi} \mathbf{e}^{b} \cdot \nabla \Phi=\frac{1}{\Phi}(\mathbf{b} \times \nabla s) \cdot \nabla \Phi \\
= & \frac{1}{\Phi B}\left[\left(\frac{\mu_{0} J}{\sigma} \nabla \theta-\frac{\mu_{0} I}{\sigma} \nabla \varphi\right) \times \nabla s\right] \cdot \nabla \Phi \\
= & \frac{1}{\Phi \sigma B}\left[\mu_{0} J(\nabla \theta \times \nabla s)-\mu_{0} I(\nabla \varphi \times \nabla s)\right] \\
& \times\left[\frac{\partial \Phi}{\partial s} \nabla s+\frac{\partial \Phi}{\partial \theta} \nabla \theta+\frac{\partial \Phi}{\partial \varphi} \nabla \varphi\right] \\
= & \frac{1}{\Phi \sigma B}(\mu_{0} J \underbrace{(\nabla \theta \times \nabla s) \cdot \nabla \varphi} \frac{\partial \Phi}{\partial \varphi} \\
& \left.-\mu_{0} I(\nabla \varphi \times \nabla s) \cdot \nabla \theta \frac{\partial \Phi}{\partial \theta}\right), \\
(\nabla / \sqrt{g} & \\
& \\
&
\end{aligned}
$$

$$
\begin{aligned}
k_{b} & =\sqrt{\mathcal{J}} k^{b}=-\frac{\sqrt{\mathcal{J}}}{\Phi \sigma B \sqrt{g}}\left(\mu_{0} J \frac{\partial \Phi}{\partial \varphi}+\mu_{0} I \frac{\partial \Phi}{\partial \theta}\right) \\
& \Rightarrow \quad k_{b} k^{b}=\sqrt{\mathcal{J}}\left[\frac{1}{\Phi \sigma B \sqrt{g}}\left(\mu_{0} J \frac{\partial \Phi}{\partial \varphi}+\mu_{0} I \frac{\partial \Phi}{\partial \theta}\right)\right]^{2} .
\end{aligned}
$$

Explicitly, using $\sqrt{\mathcal{J}}=1 / \mathrm{g}^{s s}$,

$$
\begin{aligned}
k_{\perp}^{2}= & g^{S S}\left(\frac{1}{\Phi} \frac{\partial \Phi}{\partial S}\right)^{2} \\
& +\frac{1}{g^{S S}}\left[\frac{1}{\Phi \sigma B \sqrt{g}}\left(\mu_{0} J \frac{\partial \Phi}{\partial \varphi}+\mu_{0} I \frac{\partial \Phi}{\partial \theta}\right)\right]^{2} .
\end{aligned}
$$

A.4. Parallel wave number $k_{\|}$

Since $\mathbf{e}_{\|}=\mathbf{e}^{\|}$, there is no difference between the co- and contravariant components:

$$
\begin{aligned}
k^{\|}= & \frac{1}{\Phi} \mathbf{e}^{\|} \cdot \nabla \Phi \\
= & \frac{1}{\Phi} \mathbf{b} \cdot \nabla \Phi=\frac{1}{\Phi B}\left[\psi^{\prime}(\nabla \varphi \times \nabla s)+\chi^{\prime}(\nabla s \times \nabla \theta)\right] \cdot \nabla \Phi \\
= & \frac{1}{\Phi B}\left[\psi^{\prime}(\nabla \varphi \times \nabla s)+\chi^{\prime}(\nabla s \times \nabla \theta)\right] \\
& \cdot\left[\frac{\partial \Phi}{\partial s} \nabla s+\frac{\partial \Phi}{\partial \theta} \nabla \theta+\frac{\partial \Phi}{\partial \varphi} \nabla \varphi\right]
\end{aligned}
$$




$$
=\frac{1}{\Phi B}[\psi^{\prime} \underbrace{(\nabla \varphi \times \nabla s) \cdot \nabla \theta}_{1 / \sqrt{g}} \frac{\partial \Phi}{\partial \theta}+\chi^{\prime} \underbrace{(\nabla s \times \nabla \theta) \cdot \nabla \varphi}_{1 / \sqrt{g}} \frac{\partial \Phi}{\partial \varphi}] .
$$

That is $\left(k_{\|}=k^{\|}\right)$,

$k_{\|}=\frac{1}{\Phi B \sqrt{g}}\left(\psi^{\prime} \frac{\partial \Phi}{\partial \theta}+\chi^{\prime} \frac{\partial \Phi}{\partial \varphi}\right)$.

\section{Appendix B. Relations for creating new inputs after each iteration}

The changing inputs needed by the equilibrium code VMEC are $T_{\perp} / T_{\|}(s), p_{h}(s)$, and the toroidal current $J(s)$. For LEMan we need to reconstruct the density amplitude factor $\mathcal{N}(s)$ and parallel temperature $T_{\|}(s)$ profiles. The toroidal current is directly added to the ohmic current, and no further relations are needed.

For finding the anisotropy, we invert the following relation:

$\frac{p_{\perp}^{h}(s, \theta)}{p_{\|}^{h}(s, \theta)}=M(s, A, \theta)$,

where we wrote $A=T_{\perp} / T_{\|}$for simplicity and

$M(s, A, \theta)=\left\{\begin{array}{l}A\left[\frac{B}{B_{c}}+\frac{p_{\perp}}{p_{\|}}\left(1-\frac{B}{B_{c}}\right)\right], \\ B>B_{c}, \\ A \frac{B}{B_{c}} \frac{\left[1+A\left(1-B / B_{c}\right)\right]^{2}-5\left[A\left(1-B / B_{c}\right)\right]^{3 / 2}+\left[A\left(1-B / B_{c}\right)\right]^{7 / 2}}{\left\{1-A^{2}\left(1-B / B_{C}\right)^{2}\right\}\left\{1+A\left(1-B / B_{c}\right)-2\left[A\left(1-B / B_{c}\right)\right]^{5 / 2}\right\}} \\ B<B_{C} .\end{array}\right.$

$p_{\perp}^{h}(s, \theta)$ and $p_{\|}^{h}(s, \theta)$ are known from the distribution function and $B(s, \theta)$ and $B_{c}$ are known from the equilibrium. For the regions where $B>B_{c}$, the anisotropy $A$ can directly be computed, whereas a root finding algorithm has to be applied in the case $B<B_{c}$. In VENUS a simple secant method is implemented to that end. The hot parallel pressure amplitude $p_{h}(s)$ is defined through $p_{\|}^{h}(s, \theta)=p_{t h}(s) p_{h}(s) H(s, \theta)$, where $p_{\|}^{h}(s, \theta)$ is coming from the hot distribution function, $p_{t h}(s)$ is the background pressure and $H(s, \theta)$ is defined as

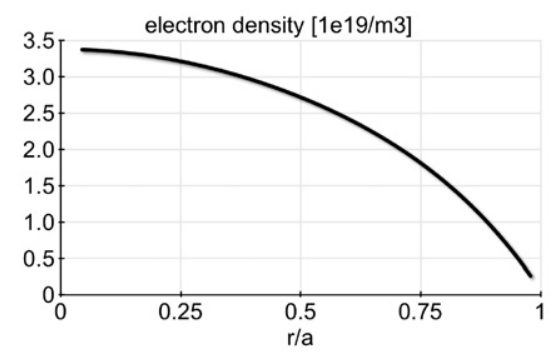

(a)

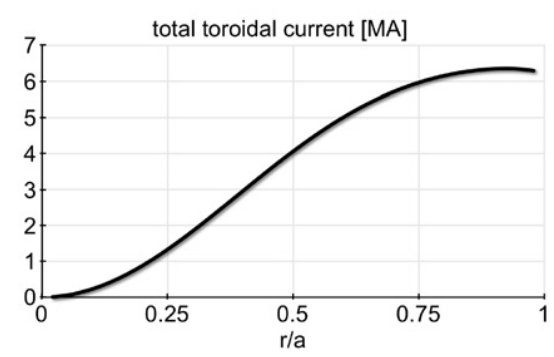

(c)
$H(s, B)=\left\{\begin{array}{l}\frac{B / B_{c}}{1-T_{\perp} / T_{\|}\left(1-B / B_{c}\right)}, \\ B>B_{c}, \\ \frac{B}{B_{c}} \frac{1+T_{\perp} / T_{\|}\left(1-B / B_{c}\right)-2\left[T_{\perp} / T_{\|}\left(1-B / B_{c}\right)\right]^{5 / 2}}{1-\left[T_{\perp} / T_{\|}\left(1-B / B_{c}\right)\right]^{2}}, \\ B<B_{c} .\end{array}\right.$

We can then write

$p_{h}(s)=\frac{p_{\|}^{h}(s, \theta)}{p_{t h}(s) H(s, \theta)}$.

For the density-like amplitude factor $\mathcal{N}(s)$, recall that $\mathcal{N}(s)$ is related to the real density by

$n_{h}(s, \theta)=\mathcal{N}(s) \sqrt{\frac{T_{\|}}{T_{\perp}}} \mathcal{C}(s, \theta)$,

with

$\mathcal{C}(s, B)=\left\{\begin{array}{l}\frac{B / B_{c}}{1-T_{\perp} / T_{\|}\left(1-B / B_{c}\right)}, \\ B>B_{c} \\ \frac{B}{B_{c}} \frac{1+T_{\perp} / T_{\|}\left(1-B / B_{c}\right)-2\left[T_{\perp} / T_{\|}\left(1-B / B_{c}\right)\right]^{3 / 2}}{1-\left[T_{\perp} / T_{\|}\left(1-B / B_{c}\right)\right]^{2}}, \\ B<B_{c} .\end{array}\right.$

Once the anisotropy is found, $\mathcal{N}$ can be determined using

$\mathcal{N}(s)=\sqrt{A(s)} \frac{n_{h}(s, \theta)}{\mathcal{C}(s, \theta)}$.

The hot parallel temperature $T_{\|}(s)$ is finally found with $p_{\|}^{h}(s, \theta)=$ $\mathcal{N}(s) T_{\|}^{h}(s) H(s, \theta)$, and thus

$T_{\|}^{h}(s)=\frac{p_{\|}^{h}(s, \theta)}{\mathcal{N}(s) H(s, \theta)}$.

Eqs. (B.1), (B.3), (B.6) and (B.7) are implemented in VENUS for finding new inputs to VMEC and LEMan at the end of each iteration.

\section{Appendix C. Equilibrium for Section 4}

$1 \%$ helium-3 in deuterium background (see Fig. 16). Major radius on axis $R_{0}=2.99 \mathrm{~m}$, maximum minor radius $a=1.17 \mathrm{~m}$,

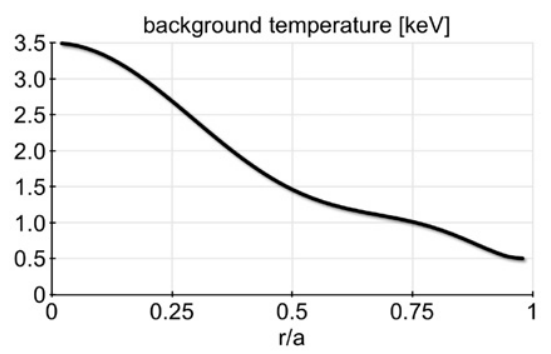

(b)

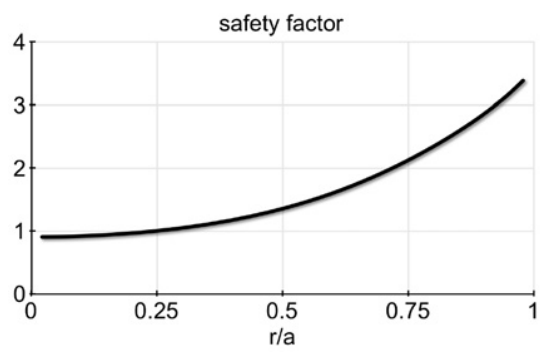

(d)

Fig. 16. Background profiles. The $\mathrm{He}^{3}$ minority initially has the same temperature and density profiles, the latter scaled by a factor of $1 \%$. The heating is applied at $r / a=0.25$. (a) Background density. (b) Background temperature. (c) Ohmic toroidal current. (d) Safety factor. 
elongation $\kappa_{a}=1.4$, triangularity $\delta_{a}=0.4, B_{0}=2.94 \mathrm{~T}$. The temperature on axis is $T_{0}^{e}=T_{0}^{i}=3.5 \mathrm{keV}$ and the density $n_{0}^{i}=0.98 n_{0}^{e}$, $n_{0}^{e}=3.38 \times 10^{+19} \mathrm{~m}^{-3}$. The resonant layer is chosen at $r / a=0.25$. This gives for the wave field $B_{c}=3.18 \mathrm{~T}$, yielding $f_{R F}=32.4 \mathrm{MHz}$.

\section{References}

[1] J.P. Graves, et al., Nucl. Fusion 50 (2010) 052002.

[2] T. Hellsten, J. Carlsson, L.-G. Eriksson, J. Hedin, A. Jaun, Plasma Phys. Control. Fusion 40 (1998) 1085

[3] M. Brambilla, R. Bilbato, Nucl. Fusion 46 (2006) S387.

[4] M. Brambilla, R. Bilbato, Nucl. Fusion 49 (2009) 085004

[5] M. Choi, et al., Phys. Plasmas 17 (2010) 056102.

[6] W.A. Cooper, et al., Nucl. Fusion 46 (2006) 683.

[7] P. Popovich, W.A. Cooper, L. Villard, Comp. Phys. Comm. 175 (2006) 250.

[8] N. Mellet, W.A. Cooper, P. Popovich, L. Villard, S. Brunner, Convolution and iterative methods applied to low-frequency waves in 3D warm configurations, Comp. Phys. Comm. 182 (3) (2011) 570-589.

[9] O. Fischer, W.A. Cooper, M.Y. Isaev, L. Villard, Nucl. Fusion 42 (2002) 817.

[10] G.A. Cooper, M. Jucker, W.A. Cooper, J.P. Graves, M.Y. Isaev, Phys. Plasmas 14 (2007) 102506.
[11] S.P. Hirshman, O. Betancourt, J. Comp. Phys. 96 (1991) 99

[12] R.J. Dumont, C.K. Phillips, D.N. Smithe, Phys. Plasmas 12 (2005) 042508.

[13] R.O. Dendy, R.J. Hastie, K.G. McClements, T.J. Martin, Phys. Plasmas 2 (1995) 1623.

[14] N. Mellet, et al., Comp. Phys. Comm. 182 (2011) 570.

[15] S. Brunner, J. Vaclavik, Phys. Fluids B 5 (1993) 1695.

[16] T. Martin, J. Vaclavik, Helvetica Physica Acta 60 (1987) 471.

[17] W.A. Cooper, J.P. Graves, M. Jucker, M.Y. Isaev, Phys. Plasmas 13 (2006) 092501.

[18] L.-G. Eriksson, M. Schneider, Phys. Plasmas 12 (2005) 072524.

[19] T.H. Stix, Nucl. Fusion 15 (1975) 737.

[20] J. Wesson, Tokamaks, 3rd edition, Oxford University Press, Great Clarendon Street, Oxford OX2 6DP, 2004.

[21] A.H. Boozer, G. Kuo-Petravic, Phys. Fluids 24 (1981) 851.

[22] A.N. Kaufman, Phys. Fluids 15 (1972) 1063.

[23] S. Murakami, et al., Nucl. Fusion 46 (2006) S425.

[24] T. Johnson, T. Hellsten, L.-G. Eriksson, Nucl. Fusion 46 (2006) S433.

[25] T. Hellsten, K. Holmström, T. Johnson, T. Bergkvist, M. Laxåbäck, Nucl. Fusion 46 (2006) S442.

[26] C.F. Kennel, F. Engelmann, Phys. Fluids 9 (1966) 2377.

[27] T.H. Stix, Waves in Plasmas, American Institute of Physics, New York, 1992.

[28] L. Chen, J. Vaclavik, G.W. Hammett, Nucl. Fusion 28 (1988) 389.

[29] L.-G. Eriksson, et al., Phys. Rev. Lett. 81 (1998) 1231. 